\title{
Lipid accumulation in human breast cancer cells injured by iron depletors
}

\author{
Maida De Bortoli ${ }^{1 \dagger}$, Elena Taverna ${ }^{1 \dagger}$, Elisa Maffioli ${ }^{2}$, Patrizia Casalini ${ }^{3}$, Francesco Crisafi ${ }^{4}$, Vikas Kumar ${ }^{4}$, \\ Claudio Caccia ${ }^{5}$, Dario Polli, ${ }^{4,6}$, Gabriella Tedeschi ${ }^{2,7}$ and Italia Bongarzone ${ }^{1 *}$ (D)
}

\begin{abstract}
Background: Current insights into the effects of iron deficiency in tumour cells are not commensurate with the importance of iron in cell metabolism. Studies have predominantly focused on the effects of oxygen or glucose scarcity in tumour cells, while attributing insufficient emphasis to the inadequate supply of iron in hypoxic regions. Cellular responses to iron deficiency and hypoxia are interlinked and may strongly affect tumour metabolism.

Methods: We examined the morphological, proteomic, and metabolic effects induced by two iron chelators - deferoxamine (DFO) and di-2-pyridylketone 4,4-dimethyl-3-thiosemicarbazone (Dp44mT)—on MDA-MB-231 and MDA-MB-157 breast cancer cells.

Results: These chelators induced a cytoplasmic massive vacuolation and accumulation of lipid droplets (LDs), eventually followed by implosive, non-autophagic, and non-apoptotic death similar to methuosis. Vacuoles and LDs are generated by expansion of the endoplasmic reticulum (ER) based on extracellular fluid import, which includes unsaturated fatty acids that accumulate in LDs. Typical physiological phenomena associated with hypoxia are observed, such as inhibition of translation, mitochondrial dysfunction, and metabolic remodelling. These survival-oriented changes are associated with a greater expression of epithelial/mesenchymal transcription markers.

Conclusions: Iron starvation induces a hypoxia-like program able to scavenge nutrients from the extracellular environment, and cells assume a hypertrophic phenotype. Such survival strategy is accompanied by the ERdependent massive cytoplasmic vacuolization, mitochondrial dysfunctions, and LD accumulation and then evolves into cell death. LDs containing a greater proportion of unsaturated lipids are released as a consequence of cell death. The consequence of the disruption of iron metabolism in tumour tissue and the effects of LDs on intercellular communication, cancer-inflammation axis, and immunity remain to be explored. Considering the potential benefits, these are crucial subjects for future mechanistic and clinical studies.
\end{abstract}

Keywords: Breast cancer, cytoplasm vacuolation, endoplasmic reticulum stress, iron chelation, lipid droplets, macropinocytosis, mitochondria dysfunctions, methuotic cell death, hypoxia, Raman spectroscopy

\section{Background}

Iron is an essential component of several cellular enzymes, such as catalases, peroxidases, cytochromes, ribonucleotide reductase, desaturases, and aconitase, which are crucial for physiological functions and have been implicated in several diseases, including cancer, because of alterations in iron metabolism [1]. Adequate iron supply is critical for various cellular processes, including DNA synthesis and cell cycle

\footnotetext{
* Correspondence: Italia.bongarzone@istitutotumori.mi.it

${ }^{\dagger}$ Equal contributors

${ }^{1}$ Fondazione IRCCS Istituto Nazionale dei Tumori, via G. Amadeo 42, Milan 20133, Italy

Full list of author information is available at the end of the article
}

progression. Many in vitro and in vivo studies have demonstrated that compared with normal cells, cancer cells are more sensitive to iron deprivation because of their marked Fe requirements [2]. Furthermore, their strong dependency on iron is evidenced by their increased expression of transferrin receptors compared with that of normal cells. In vitro and animal studies have also indicated the antitumour activity of several iron chelators [3-5].

Clinical data support the concept that iron deficiency increases angiogenesis and causes breast cancer recurrence [6]. Specifically, iron deficiency can contribute to the high recurrence of breast cancer in premenopausal women, 
whereas iron load might play a role in the metastasization of breast cancer in postmenopausal women.

Iron chelators are known to induce apoptosis in several types of proliferating cells [7] and are therefore considered promising anti-proliferative agents in the treatment of human cancers. Iron chelators, such as deferoxamine (DFO), deferiprone, and deferasirox, have several advantages: they have been clinically approved for iron overload disorders [8], they have a well-studied, long-term use toxicity profile, and experimental results could be readily translated into clinical trials for cancer. Nevertheless, it is crucial to consider several side effects related to their use, including myelosuppression, hypoxia, and methemoglobinaemia, as observed from clinical trials [9].

DFO is a clinically approved non-toxic iron chelator that has been effectively used for long-term iron chelation therapy in beta-thalassemia and other iron overload disorders. DFO has also been reported to have some antitumour activity [10-13]. Novel chelators based on the di-2-pyridylketone thiosemicarbazone (DpT) scaffold, such as di-2-pyridylketone 4,4-dimethyl-3-thiosemicarbazone (Dp44mT), induce iron sequestration and also form redox-active metal complexes that demonstrate potent and selective antitumour activity [14]. Notably, $\mathrm{Dp} 44 \mathrm{mT}$ and its analogues possess broad anti-cancer and anti-metastatic activity, in vitro and in vivo, against several aggressive solid tumours [15-20].

Relevant studies have focused on the causality between iron chelation and cancer cell death, without considering that substantial regions of cancers often grow in hypoxic conditions owing to the lack of a functional vasculature. The amount of bio-available iron is often limited in poorly vascularised areas, and the iron uptake in cancer cells is inadequate to fulfil their need. Moreover, the lack of cellular iron content has an essential role in positively regulating hypoxia-inducible factor (HIF) protein stability and therefore hypoxia mechanisms, even under non-hypoxic conditions [21]. Thus, cancer cells are more susceptible to iron depletion than non-cancer cells, a phenomenon we have termed iron addiction; however, it is important to note that cancer cells adapt in response to low iron levels, directly affecting cancer cell metabolism [22]. Recent findings on medulloblastoma cell lines indicate that modulation of iron-related proteins during hypoxia may increase cell proliferation as well as tumour aggression and stemness [23]. Oxygen and iron are intimately linked in producing signals through the hypoxia response pathway, and they exert considerable influence on cancer cell metabolism [24].

Based on these observations, we aimed to understand the morphological, proteomic, and metabolic effects of iron depletion in breast cancer cells with a deeper insight into the cellular effects and drawbacks of iron starvation. We combined biochemistry, microscopy, flow cytometry, and mass spectrometry-based methods to investigate the cellular and molecular events induced by DFO or $\mathrm{Dp} 44 \mathrm{mT}$ in two human breast cancer cell lines, MDAMB-231 and MDA-MB-157.

Cellular stress and organelle dysfunctions due to the disruption of cellular homeostasis were observed, and these events led to cell death. A dramatic increase in endoplasmic reticulum (ER) complexity, with the appearance of large vacuoles and the accumulation of lipid droplets (LDs), accompanied mitochondrial dysfunction and bioenergetics collapse and was followed by cell death and LD leakage. The results of this study are expected to provide important insights into the fundamental molecular mechanisms of the adaptation of breast cancer cells to iron limitation.

\section{Methods}

Cell lines and culture

MDA-MB-231 and MDA-MB-157 breast cancer cell lines were obtained from the American Type Culture Collection (ATCC, Manassas, VA, USA). They are characterised as triple-negative/basal B mammary carcinoma and considered models of triple-negative breast cancer growth and progression. Cell lines were maintained in Dulbecco's modified Eagle's medium (DMEM; Gibco) containing $10 \%$ heat-inactivated foetal bovine serum (FBS; HyClone), DMEM w/ $4.5 \mathrm{~g} / \mathrm{L}$ glucose w/o Lglutamine at $37{ }^{\circ} \mathrm{C}$ and $5 \% \mathrm{CO}_{2}$ in air.

\section{Reagents}

Dp44mT and DFO were purchased from Sigma-Aldrich. Dp44mT was dissolved in dimethyl sulfoxide (DMSO) and further diluted to a final concentration of $5 \mu \mathrm{M}$ in the culture medium, whereas DFO was diluted to a final concentration of $250 \mu \mathrm{M}$ in the culture medium and used at a final concentration of $100 \mu \mathrm{M}$. Cells were incubated with either control media containing or not containing DMSO at $0.05 \%(v / v)$ to match the concentration of the dissolved Dp44mT or DFO.

\section{Assessment of antiproliferative activity}

3-[4,5-dimethylthiazol-2-yl]-2,5-diphenyltetrazolium bromide thiazolyl blue (MTT) assay was performed in 48multiwell plates containing MDA-MB-231 cells, seeded at a concentration of $1.8 \times 10^{4}$ cells per well. After $24 \mathrm{~h}$, MDAMB-231 cells were treated with $5 \mu \mathrm{M}$ Dp44mT or with $100 \mu \mathrm{M}$ DFO for $120 \mathrm{~h}$. Cell proliferation was assessed by MTT (3-[4,5-dimethylthiazol-2-yl]-2,5-diphenyltetrazolium bromide; thiazolyl blue) assay according to the manufacturer's recommendations (Sigma). Absorbance intensity was quantified at $492 \mathrm{~nm}$ using a microplate reader (Infinite 200 , Tecan). Data are shown as mean \pm standard error of the mean (SEM) of quadruplicated wells and are representative of three independent experiments. Statistical tests were performed using GraphPad Prism version 5.0 (GraphPad Software Inc.). 
Sample preparation, SDS-PAGE, and immunoblotting

MDA-MB-231 and MDA-MB-157 cell lines were treated with the iron chelators for the times indicated earlier. Cell pellets were solubilised as previously described [25]. Protein concentrations were detected using a bicinchoninic acid (BCA) kit (Pierce) according to the manufacturer's instructions, and SDS-PAGE and electroblotting were performed, as previously described [26]. The antibodies used for western blotting analyses are listed in Additional file 1.

\section{Mitochondrial membrane potential analysis}

After treating cells with the iron chelators for the times indicated earlier, the mitochondrial membrane potential of the cells was detected using a MitoProbe ${ }^{\mathrm{Tu}}$ JC-1 assay kit for flow cytometry (Thermo Fisher Scientific). Flow cytometry analyses were performed as previously described [27].

\section{Fluorescence microscopy}

The subcellular location of various proteins was visualised by fluorescence microscopy (Eclipse E1000, Nikon Instruments, Inc.). To visualise different subcellular compartments, we used antibodies for RAB5, RAB7, LAMP1, NDRG1, and RTN4 as markers for the ER and LysoTracker red (50 nM; Life Technologies) as a marker for lysosomes. To visualise mitochondria in live cells, we stained the cells with the vital dye Rhodamine 123 (500 ng/mL; Sigma-Aldrich) for $15 \mathrm{~min}$ at $37{ }^{\circ} \mathrm{C}$. To visualise nuclei in live cells, we stained the cells with the cell-permeable DNA dye 4',6-diamidino-2-phenylindole (DAPI) $\left(10 \mu \mathrm{M}\right.$; Molecular Probes) for $15 \mathrm{~min}$ at $37^{\circ} \mathrm{C}$.

\section{Confocal microscopy}

Confocal laser scanning microscopy was performed using the Leica TCS SP8 X microscope (Leica Microsystems $\mathrm{GmbH})$.

\section{Nano-scale LC-MS/MS analysis}

Cells were lysed in $50 \mu \mathrm{L}$ of $0.1 \%$ RapidGest SF Surfactant (Waters) and diluted in $50 \mathrm{mM}$ ammonium bicarbonate (pH 8.0). After reduction and alkylation, the proteins were digested with trypsin sequence grade (Roche) for $16 \mathrm{~h}$ at $37^{\circ}$ $\mathrm{C}$ using a protein:trypsin ratio of 20:1 [28]. LC-ESI-MS/MS analysis was performed on a Dionex UltiMate 3000 HPLC System with a PicoFrit ProteoPrep C18 column $(200 \mathrm{~mm}$ in length and with an internal diameter of $75 \mu \mathrm{m})$ (New Objective). The eluate was electrosprayed into an LTQ Orbitrap Velos (Thermo Fisher Scientific) through a Proxeon nanoelectrospray ion source (Thermo Fisher Scientific) [29]. Four technical replicate analyses of each sample were performed. Data acquisition was controlled by Xcalibur 2.0 and Tune 2 . 4 software (Thermo Fisher Scientific).

Mass spectra were analysed using the MaxQuant software (version 1.3.0.5). Enzyme specificity was set to trypsin. Carbamidomethylcysteine was set as a fixed modification, and $\mathrm{N}$-terminal acetylation, methionine oxidation, and asparagine/glutamine deamidation were set as variable modifications. The spectra were searched by the Andromeda search engine against the human Uniprot sequence database (release 29.06.2015). Protein identification required at least one unique or razor peptide per protein group. Quantification in MaxQuant was performed using the built-in XIC-based label-free quantification (LFQ) algorithm [30] using fast LFQ. The required false positive rate was set to $1 \%$ at the peptide level and $1 \%$ at the protein level. Statistical analyses [31] were performed using the Perseus software (version 1.4.0.6, www.biochem.mpg.de/mann/tools/).

Only proteins present and quantified in at least 3 out of 4 technical repeats were considered positively identified; 2478,2488 , and 2496 proteins were identified in untreated, DFO-, and Dp44mT-treated cells, respectively; 108 proteins were exclusively expressed in untreated/control cells, 54 proteins in DFO/cells, and 137 proteins in Dp44mT/cells. An ANOVA test (false discovery rate 0.05 ) was carried out to identify proteins differentially expressed among the three conditions: 1682 out of 2149 common proteins differed with statistical significance and were selected for further analyses. In particular, we focused on the differential proteomics between proteins expressed in untreated cells and proteins expressed in DFO- or Dp44mT-treated cells. Differential expression was considered significant if (1) a protein was present only in untreated or treated cells or (2) its normalised (according to the LFQ algorithm) intensity resulted in a statistical difference, as calculated by the Welch's $t$-test $(t$-test cut-off at $p$ value $=0.0167$ ). The MS proteomics data have been deposited in the ProteomeXchange Consortium via the PRIDE partner repository [32] with the dataset identifier PXD007595.

\section{Gene ontology (GO)}

The Search Tool for the Retrieval of INteracting Genes/ proteins (STRING) database (version 10.5, Database issue: D412-416) [33] was used for prediction of Kyoto Encyclopedia of Genes and Genomes (KEGG) pathways [34-36]. A GO scatterplot was constructed in Excel.

\section{Oil red $O$ staining}

To determine the presence of LD accumulation within MDA-MB-231 and MDA-MB-157 cells, Oil Red O (SigmaAldrich) staining was performed. To visualise cell nuclei, samples were stained with haematoxylin (Sigma-Aldrich). Cells were imaged on a Leica DM IRB microscope (Leica Microsystems).

\section{Fatty acid (FA) quantification in lipid droplets}

Cells were cultured in $10-\mathrm{cm}$ dishes for $96 \mathrm{~h}$ in the presence of $100 \mu \mathrm{M}$ DFO or Dp44mT. The presence of LDs was evaluated with Oil Red O staining. Cell debris was 
recovered from the plates and LDs purified by density sucrose gradient [37].

Lipids were prepared by homogenizing the samples in ethanol containing (50 ppm) butylated hydroxy toluene (BHT) to avoid oxidation [38]. A lipid chromatogram was obtained by gas chromatography-mass spectrometry (GC-MS) analysis using a Shimadzu gas chromatograph equipped with a quadrupole mass spectrometer for electron impact ionisation (GC-MS-QP2010). An SH Stabilwax DA column ( $30 \mathrm{~m}$ in length, $0.25 \mathrm{~mm}$ in diameter, and with a film thickness $0.25 \mu \mathrm{m}$ ) was used to separate the FA methyl ester at a flow rate of $1.0 \mathrm{~mL} / \mathrm{min}$. The injector temperature was set to $200^{\circ} \mathrm{C}$ and the transfer line temperature to $280^{\circ} \mathrm{C}$. The GC oven was programmed as follows: after $2 \mathrm{~min}$ at $50^{\circ} \mathrm{C}$, the temperature was increased at $30^{\circ} \mathrm{C} / \mathrm{min}$ to $150^{\circ} \mathrm{C}$, then at $15^{\circ} \mathrm{C} / \mathrm{min}$ to $230^{\circ}$ C. The total run duration was $25 \mathrm{~min}$. GC-MS analysis was conducted in the full scan mode (m/z 35-600). Qualitative analysis was based on the characteristic ions of the FA methyl esters and their relative retention times. Quantitative analysis was based on the ratio between the peak area of each FA and the corresponding internal standard peak area, using the respective standard curves.

\section{Raman spectroscopy}

To perform coherent anti-Stokes Raman scattering (CARS) imaging, a home-built laser scanning multi-modal microscope, described previously [39], was utilised. To acquire CARS images, the treated cells were placed under the laser focus of the microscope, and the laser spot was galvoscanned over the $50 \times 50 \mu \mathrm{m}^{2}$ sample area at two fixed pump-Stokes frequency detuning: $2850 \mathrm{~cm}^{-1}$ (for lipid molecules) and $3050 \mathrm{~cm}^{-1}$ (for medium and vacuoles). Spectral profiles and spatially resolved chemical maps of the pure chemical components were generated using the MATLAB tool box MCR-ALS (Multivariate Curve Resolution Alternating Least Squares) algorithm [40, 41]. We collected two images: one at $2850 \mathrm{~cm}^{-1}$, indicating the symmetric vibration of $\mathrm{CH}_{2}$ chains in lipids, and the other at $3050 \mathrm{~cm}^{-1}$, indicating the stretching vibration of hydrogen-bonded water of saline environments. The output of the software was two matrices (representing the concentration at $2850 \mathrm{~cm}^{-1}$ and $3050 \mathrm{~cm}^{-1}$ ) and two spectra (representing the two identified species). For the set-up of the figure, we have plotted these two matrices from grayscale to a single-color scale (green for lipids, blue for medium) and created a composite image thereof with the FIJI image processing package.

\section{Results}

Targeting iron homeostasis induces cell death by formation of vacuoles

We observed that the treatment of MDA-MB-231 cells with $100 \mu \mathrm{M}$ DFO or $5 \mu \mathrm{M}$ Dp44mT induced extensive cytoplasmic modifications at different time points $(24 \mathrm{~h}$, $48 \mathrm{~h}$, and $72 \mathrm{~h})$. At $48-72 \mathrm{~h}$ after treatment with $100 \mu \mathrm{M}$ DFO, the cells became flattened and showed extensive vacuolation (Fig. 1a). Cytoplasmic vacuolation began to be evident at $24 \mathrm{~h}$ after $\mathrm{Dp} 44 \mathrm{mT}$ treatment; it was more pronounced after $48 \mathrm{~h}$ and involved extensive regions of the cytoplasm at $72 \mathrm{~h}$. The MTT assay indicated that at $120 \mathrm{~h}$ after treatment with $100 \mu \mathrm{M}$ DFO, MDA-MB-231 cell proliferation was completely blocked (Fig. 1b). Treatment with $5 \mu \mathrm{M}$ Dp44mT drastically reduced cell proliferation after $48 \mathrm{~h}$ and killed all the cells within $120 \mathrm{~h}$. Evaluation of the cell cycle distribution of MDA-MB-231 cells after treatment with DFO or $\mathrm{Dp} 44 \mathrm{mT}$ showed that there was a slight increase in the G2 population (Additional file 2). For both treatments, the morphology of the cells during death could neither be classified as apoptotic nor as necrotic.

Western blot analyses revealed that DFO and Dp44mT induced a very low poly (ADP-ribose) polymerase-1 (PARP) cleavage and high mobility group box 1 (HMGB1) expression in cells after $72 \mathrm{~h}$ (Fig. 1c). B-cell lymphoma 2 (BCL-2) expression did not change significantly in response to either DFO or Dp44mT treatments. The results using biomarkers of apoptosis and necrosis indicated the irrelevant contribution of both apoptotic and necrotic cell death pathways. The levels of p-JNK and p-p38 drastically decreased after $72 \mathrm{~h}$ in untreated cells but persisted at high levels in treated cells; a slight increase in p-AMPK and p-mTOR levels was observed at $72 \mathrm{~h}$ in both untreated and treated cells. JNK and p38 signalling may be triggered by the redox staterelated perturbations and may regulate expression of apoptotic downstream genes, preventing apoptosis and promoting cell survival. These results suggest the development of stress-related compensatory mechanisms to overcome cell death and permit short-term cell survival.

\section{Proteome analyses}

We performed a proteomics analysis to investigate the effects of DFO and Dp44mT in MDA-MB-231 cells after treatment for $72 \mathrm{~h}$, when a significant change in phenotype and morphology was observed. DMSO-treated cells were used as control. Shotgun label-free quantitative analysis was performed to examine the differences in global protein expression. After data filtering, the analysis resulted in the identification of 2478 (DMSO), 2488 (DFO), and 2496 $(\mathrm{Dp} 44 \mathrm{mT})$ proteins. One hundred eight proteins were exclusively expressed in untreated/control cells, 54 proteins in $\mathrm{DFO} /$ cells, and 137 proteins in Dp44mT/cells (Additional files 3, 4, 5, 6). An ANOVA test (false discovery rate 0.05) was carried out to identify proteins differentially expressed among the three conditions: 1682 out of 2149 common proteins differed with statistical significance and were selected for further analyses. Figure 2a shows the volcano plots summarizing the analysis of differentially expressed 
a

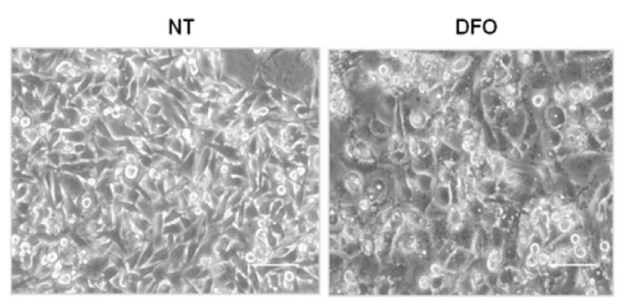

b
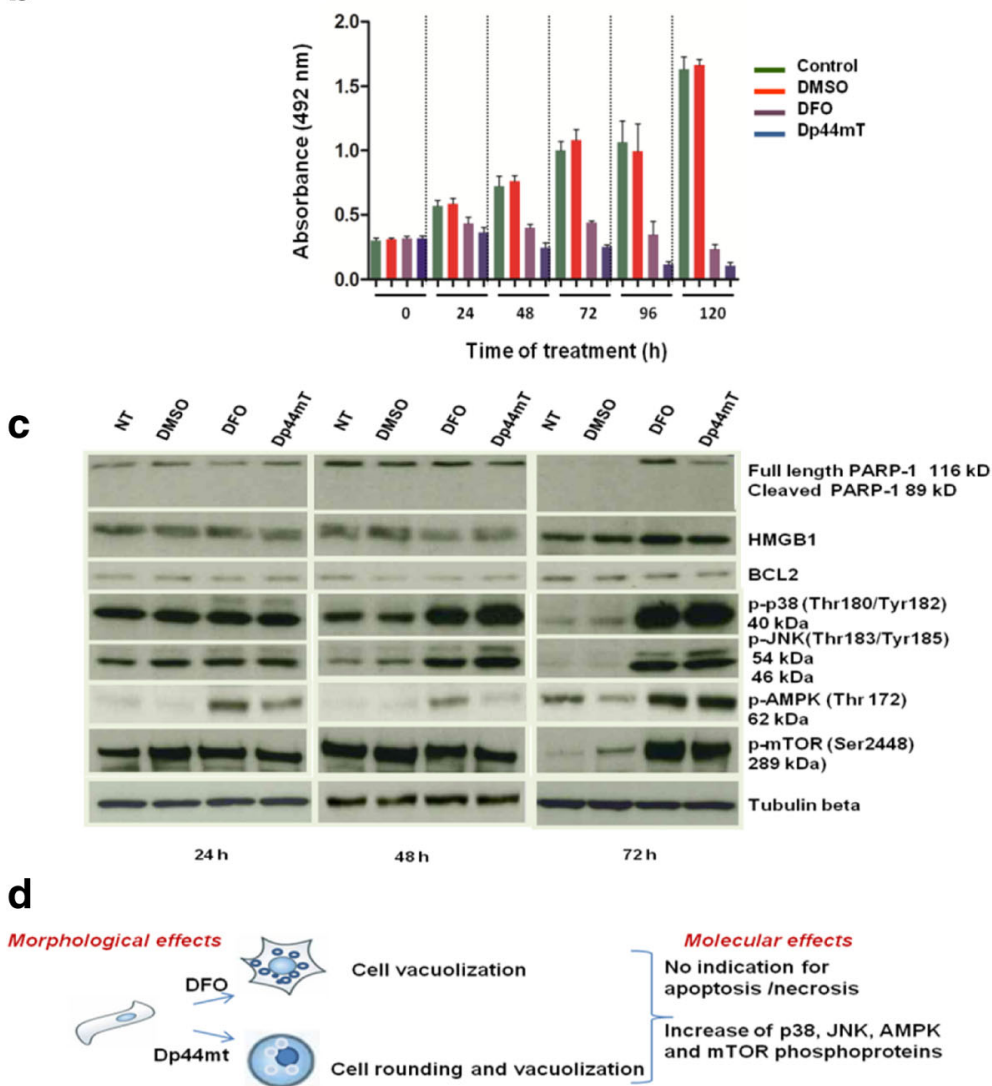

Fig. 1 DFO/Dp44mT-induced vacuolation leads to non-apoptotic/necrotic cell death in MDA-MB-231 breast cancer cells. a Representative images of MDA-MB-231 cells treated with $100 \mu \mathrm{M}$ DFO or with $5 \mu \mathrm{M}$ Dp44mT. The cells were treated with the iron chelators for $48 \mathrm{~h}$ and photographed under an optical microscope. Scale bars: $100 \mu \mathrm{m}$. b MDA-MB-231 cells treated with $100 \mu \mathrm{M}$ DFO or with $5 \mu \mathrm{M}$ Dp44mT to determine the effects of the two iron chelators on cell proliferation. An MTT assay analysis indicated that after $24 \mathrm{~h}$, cells treated with DFO or Dp44mT showed a slight decrease in cell proliferation. After Dp44mT treatment for $48 \mathrm{~h}$, cell proliferation was reduced by $69 \%$, whereas DFO treatment elicited a decrease in cell proliferation by $45 \%$. After $72 \mathrm{~h}$, Dp44mT treatment resulted in a considerable decrease in cell proliferation (75\%), whereas DFO treatment reduced cell proliferation by 56\%. Finally, after $120 \mathrm{~h}$, proliferation was effectively blocked in MDA-MB-231 cells treated with both chelators. c Determination of the expressions of PARP, HMGB1, BCL-2, phosphorylated p38, JNK, AMPK, and mTOR, following treatment of MDA-MB-231 cells with DFO or Dp44mT. Cells were treated with $100 \mu \mathrm{M}$ DFO or $5 \mu \mathrm{M}$ Dp44mT and the protein levels were examined over a period of 24-72 h. In control samples, no PARP-1 expression was observed at $72 \mathrm{~h}$. At $116 \mathrm{kDa}$ corresponding to full-length PARP-1, immunoreactivity was detectable in the 24, 48, and $72 \mathrm{~h}$-treated samples. No cleavage products of PARP-1 were seen at $48 \mathrm{~h}$ and $72 \mathrm{~h}$. Weak increase in HMGB1 expression was noted in treated cells after $72 \mathrm{~h}$. No change in the protein levels of BCL-2 was observed. The increased levels of phosphorylated p38, JNK, AMPK, and mTOR suggest that DFO and Dp44mT induce cell death by maintaining multiple activated pathways. Tubulin was used as a loading control. $\mathbf{d}$ Schematic representation of the effects observed with DFO or Dp44mT treatments

proteins in untreated cells in comparison with proteins expressed in DFO- or Dp44mT-treated cells.

The differentially expressed proteins for both treatments, identified in the label-free MS data, were unified and subjected to the analysis of the most significant KEGG pathways [35] using STRING software version 10 [33]. The most statistically significant decreased pathway identified by KEGG enrichment was Ribosome ( $\# 03010$; $p$-value $=3$. $57 \times 10^{-25}$ ) with 38 proteins (Fig. $2 b$ ). However, to reflect a multi-functional stress response, several statistically significant changes in key processes and pathways were observed, referable to enhanced glycolysis, reduced translation, 
a

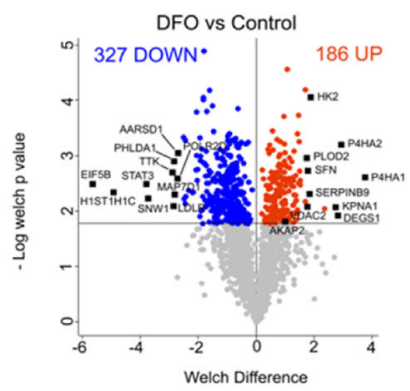

b

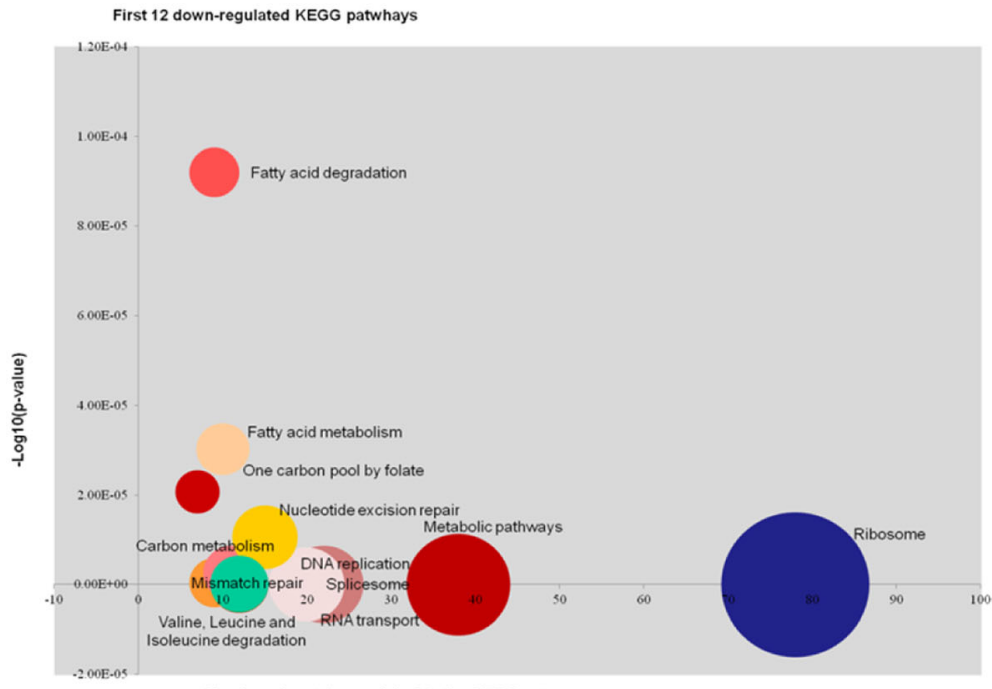

Number of proteins enriched in the KEGG patways

C

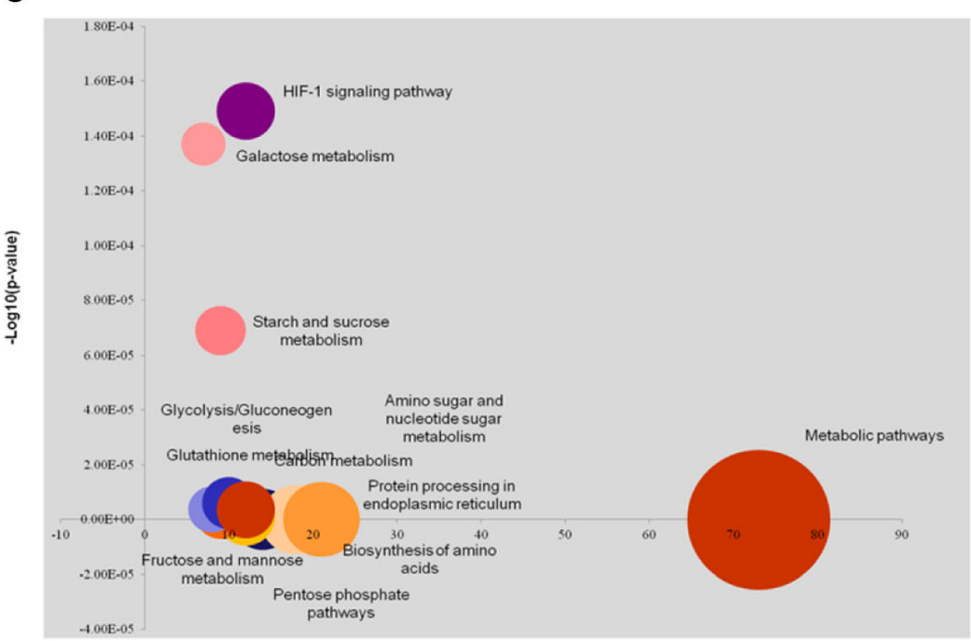

Number of proteins enriched in the KEGG patways
DpT44mT vs Control

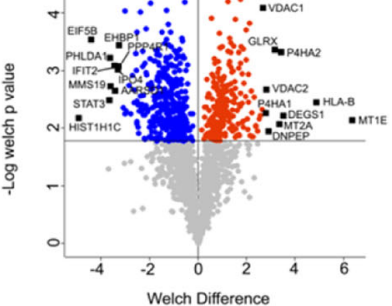

Welch Difference 396 DOWN $. \quad: \quad 237$ UP 
reduced glucose entry into the tricarboxylic acid (TCA) cycle, up-regulated glutathione metabolism and glutaminolysis, and changes in mRNA, amide, and lipid metabolism. Significant increases of the amino acid metabolism also took place as well as reduction-oxidation (redox) homeostasis and NAD metabolism. Taken together, these changes indicated a metabolic adaptation similar to that sustaining cancer cell survival during hypoxia [42].

Interrogation of the Ingenuity Pathway Analysis software highlighted that the canonical and toxicity pathways were altered by DFO or Dp44mT (Additional file 7). The analysis of pathways, conducted by combining all the up- and down-regulated proteins, indicated that EIF2 signalling, eIF4-p70S6K signalling, mTOR signalling, oxidative stress, glycolysis, gluconeogenesis, and protein degradation mediated by ubiquitination were the most common and important pathways affected by DFO and Dp44mT. Tox list analysis indicated that renal necrosis/cell death pathway, mitochondrial dysfunction, HIF signalling, oxidative stress, protein degradation mediated by ubiquitination, and fatty acid (FA) metabolism were the most affected pathways.

The observed changes can be largely explained by the induction of severe defects in the $\mathrm{Fe}-\mathrm{S}$ cluster biogenesis/repair, which can profoundly decrease the activities of respiratory complexes, ribosome biogenesis, and the TCA cycle, affecting energy production and increasing oxidative stress. The defects in the $\mathrm{Fe}-\mathrm{S}$ cluster biogenesis might also affect cytosolic aconitase activity and therefore citrate metabolism, which can disrupt the balance between glycolysis, FA biosynthesis, and purine catabolism pathways. An increase in glutamic-oxaloacetic transaminase (GOT) 1 activity was observed, indicating a possible increase in glutamine (Gln) metabolism with an apparently high uptake of Gln through up-regulation of sodium-dependent neutral amino acid transporter type 2 (SLC1A5). Along with the metabolic modifications in the treated cells, we also regarded the reduction of FA synthase (FASN) as possibly indicative of the reduction of lipid synthesis; the up-regulation of perilipin (PLIN) 2 and PLIN3 was predictive of LD accumulation [43] and the variation in desaturase levels (decreases in FA desaturase (FADS) 2 [44] and increases in D4dihydroceramide (DhCer) desaturase (DEGS) 1 [45]).

The KEGG list of up-regulated pathways included the amino sugar and nucleotide sugar metabolic pathways (Fig. $2 \mathrm{~b}$ and $\mathrm{c}$ ). The hexosamine biosynthetic pathway (HBP) is frequently up-regulated in cancer cells with a mesenchymal phenotype [46]. It is required to sustain sufficient growth factor signalling and Gln uptake to support cell growth and survival. Glucose and Gln are both essential for the first committed step and rate-limiting step of the HBP: the conversion of fructose-6-phosphate (Fru-6P) to glucosamine-6phosphate is required for the glycosylation of the proteins controlling the O-GlcNAcylation of nuclear and cytoplasmic proteins. Hypoxic cancer cells have been shown to contain elevated levels of the HBP genes, including Gln-fructose-6phosphate transaminase 1 (GFPT1) (which was overexpressed in our study) and GFPT2, and overall OGlcNAcylation [47]. Taken together, these data support a model in which by inducing a hypoxia-like metabolic reprogramming, iron chelation could solicit an HBP-based survival program that can help establish a short-term survival strategy in MDA-MB-231 cells to avoid apoptosis.

\section{Blocking of autophagy}

In principle, iron depletion can induce the activation of pathways linked to the activation of autophagy. The vacuoles formed in response to iron depletion in this study were similar to the lysosomal structures reported in several autophagic circumstances [48-52]. Thus, we investigated the possibility that the vacuoles observed in our study were also associated with autophagy. Microtubule-associated protein light chain 3 (LC3) and other members of its family are retained inside autophagosomes. Thus, LC3 is commonly used as a marker for identifying autophagosomes [53-56]. Positivity for LC3 punctae increased with both DFO and Dp44mT treatments; however, LC3 was also present in untreated cells (Fig. 3a, b). These data were further confirmed by western blotting analysis, with only a slight increase in LC3-II lipidated isoforms in treated cells (Fig. 3c).

We investigated lysosomal conditions in MDA-MB-231 cells treated with DFO or Dp44mT using lysosomotropic metachromatic fluorochrome (LysoTracker) that emits a red fluorescence at high concentrations within intact lysosomes [57]. Quantitative data from flow cytometry indicated that fluorescence increased after DFO and Dp44mT treatments (Fig. 3d). The cells exposed to Dp44mT accumulated LysoTracker in the cytoplasm, indicating a certain amount of lysosomal membrane permeabilisation. Control cells and DFO-treated cells displayed a discrete punctate fluorescent staining pattern in perinuclear regions with no fluorescence in the cytoplasm, whereas Dp44mT-treated cells displayed vacuole staining and some membrane leakage, as indicated by the diffusion of fluorescence into the cytoplasm. However, the LysoTracker staining merged with LC3 staining only partially.

Lucifer Yellow (LY) is a marker of fluid-phase pinocytosis that accumulates in secondary lysosomes and cellular membranes are impermeable to LY [58]. We loaded MDAMB-231 cells treated for $48 \mathrm{~h}$ with DFO or Dp44mT with $\mathrm{LY}$ in the final $16 \mathrm{~h}$ (Fig. 3e). Control cells and DFO-treated cells had clear punctuate labelling, possibly corresponding to the lysosomal vesicles; however, the large vacuoles did not fluoresce or fluoresced scarcely. Dp44mT-treated cells also had scarce LY-positive punctate vesicles, but often, the fluorescence was more diffuse throughout the cell body, indicating leakage of LY from the secondary lysosome into the cytosol. 


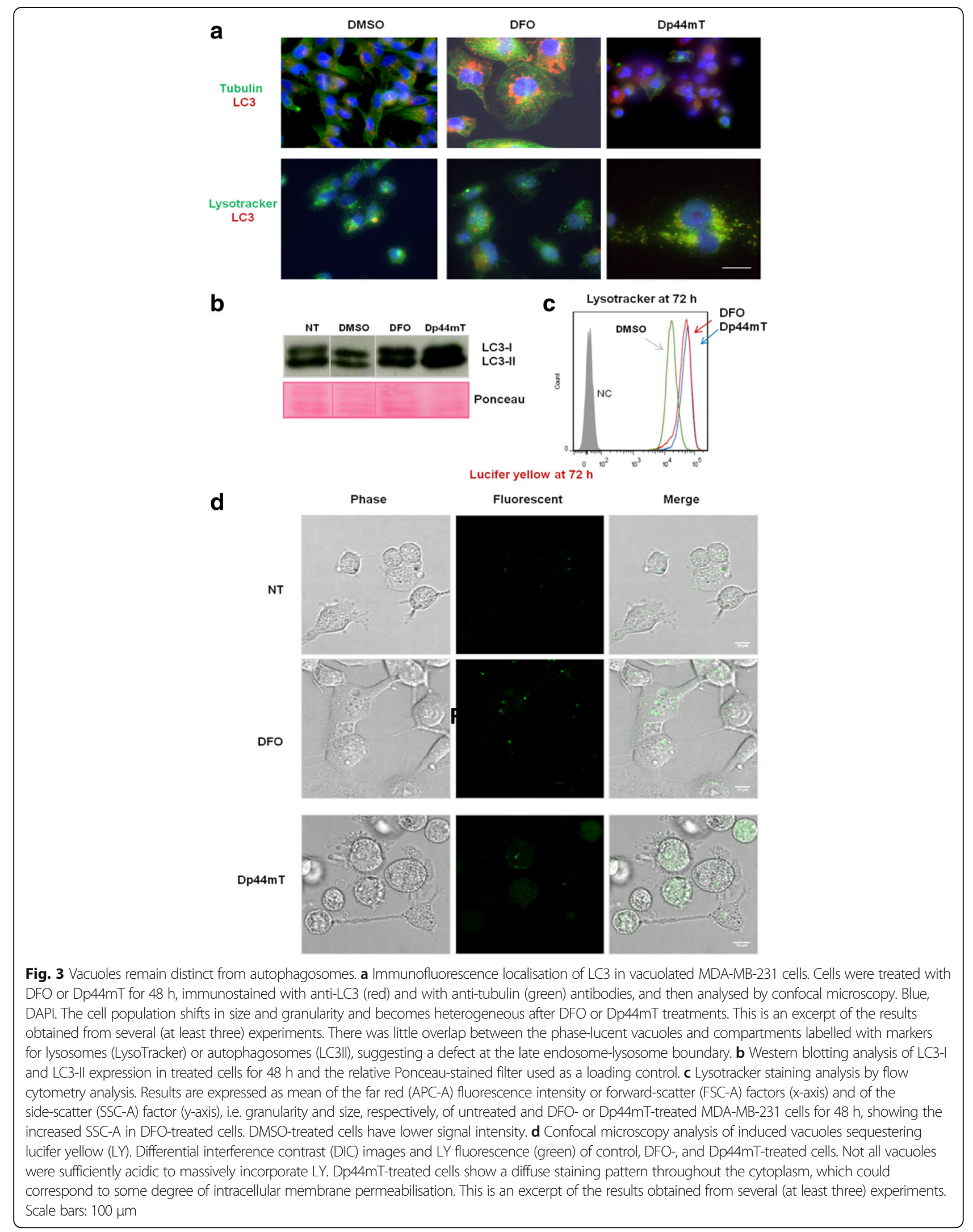


These findings strongly suggest that the vacuoles do not merge with lysosomes or autophagosomes and do not have acidic content. It was clear that most vacuoles were initially derived from another source, and one possibility was that they originated from non-functional late endosomes.

\section{Iron depletion-induced vacuolisation of cells results from ER dilation}

Immunofluorescence analysis for the lysosomal-associated membrane protein (LAMP) 1 showed an increase in total LAMP1 labelling in treated cells (Fig. 4a). Vacuoles were partially covered by LAMP1. In immunofluorescence microscopy experiments, DFO- and Dp44mT-induced vacuoles in MDA-MB-231 cells were well distinguished using anti-tubulin and anti-RAB 7 antibodies (Fig. 4b). Proteomics analysis showed that both tubulin and RAB 7 were up-regulated (Additional files 4, 6). Additionally, the endosomal marker, transferrin receptor (TfR), was also upregulated by DFO and Dp44mT treatments, as determined by proteomics analysis (Additional files 4,6 ) and immunofluorescence microscopy experiments (Fig. 4).

These results indicated that vacuoles represented enlarged late endosomal structures. Large intracellular vacuoles are membranous structures derived from intracellular organelles that are frequently formed in response to cell stress [59]. These observations suggested a possible, previously unidentified link between ER expansion and the adverse effects of DFO and Dp44mT.

$\mathrm{N}$-myc downstream-regulated gene 1 (NDRG1) is a stress-responsive protein [60]. Immunofluorescence microscopy and western blotting data showed an increased expression of the metastasis suppressor, NDRG1, after treatment with DFO or Dp44mT. NDRG1 was diffusely expressed in the cytoplasm and nucleus in untreated cells; the treatments caused the redistribution of NDRG1 with a punctate deposition around the vacuoles. NDRG1 partly co-localised with cytoplasmic and perinuclear tubulin (Fig. $4 \mathrm{c})$, which is in accordance with previous results [61], but contradicts an earlier interpretation that epithelial-mesenchymal transition (EMT) is paralleled by a downregulation of NDRG1 expression [62]. Along with NDRG1 overexpression, we observed the loss of cell-cell junctions and gain of mesenchymal characteristics via overexpression of the mesenchymal marker, vimentin [63], and decreased expression of the epithelial marker, beta-catenin (Fig. 4b).

To gain insights into the diversity of vacuolar structures formed from the expansion of the ER-for instance, if they are flat and cisternal or highly curved and tubular-we examined the distribution of reticulon 4 (RTN4), an ERshaping protein, that was observed to be up-regulated in our proteomics analysis (Additional files 4, 6). Figure 4c illustrates the sharp demarcation of the tubule-to-vacuole change at the cell periphery, as displayed by immunostaining of
RTN4. A relatively planar network could be stained in the thin periphery of the cells, but a dense ER-associated intensity was seen in the perinuclear regions with the increase in vertical space. This pattern has been described as providing an increased surface for modulating lipid synthesis or protein folding [64]. Such a tubular membrane reservoir may also be needed to facilitate the availability of ER membranes for modulating interactions with other organelles and intracellular entities, such as mitochondria, LDs, or endocytic compartments [64].

Following the proteomics analysis, we noted a strong increase in PLINs 2 and 3 (Additional files 4, 6), which are considered LD scaffold proteins that serve as critical determinants of LD formation and cellular lipid metabolism within mammalian cells $[65,66]$. In contrast, septins regulate the fluid-phase cargo traffic to lysosomes by promoting macropinosome maturation, and the fusion with endosomes/lysosomes were down-regulated (Additional files 3, 5).

\section{Disruption of mitochondrial membrane potential $(\Delta \psi \mathrm{m})$}

Mitochondria perform important catabolic reactions, such as the Krebs cycle and oxidative phosphorylation, which enable efficient ATP generation required for many cell functions. Mitochondria physically interact with the ER, and this interaction is important for both lipid synthesis and calcium handling [67]. During nutrient starvation, mitochondria elongate in order to preserve their metabolic function. To determine possible changes in mitochondrial distribution associated with ER expansion, we performed immunostaining experiments with Rhodamine 123. The Rhodamine 123 staining pattern clearly indicated a change in the mitochondrial network morphology after treatment with both chelators (Fig. 5a), possibly predicting the blockage of mitochondrial fission. In untreated cells, mitochondria were distributed throughout the cytosol, with a tendency to aggregate around the nucleus, forming a network. Following DFO or Dp44mT treatment, we observed fragmented mitochondria around ER vacuolar structures. Anti-VDAC1 staining confirmed this mitochondrial pathway (Fig. 5c). DFO and Dp44mT caused significant network fragmentation that typically occurs in response to severe stress, impaired oxidative phosphorylation, and cellular dysfunction [68].

The change in Rhodamine 123 fluorescence intensity reflects the change in relative levels of $\Delta \Psi \mathrm{m}$ or reactive oxygen species (ROS); consistent changes were observed in both treatments but with varying degrees. Both treatments produced a bi-parametric population represented by the intensity of the signals corresponding to the $x$ axis evaluated as a function of time (Fig. 5d). Mean fluorescent intensity of the treated cells decreased with respect to that of untreated cells.

The expression of the mitochondrial marker SRA stemloop interacting RNA-binding protein (SLIRP) decreased at 
a

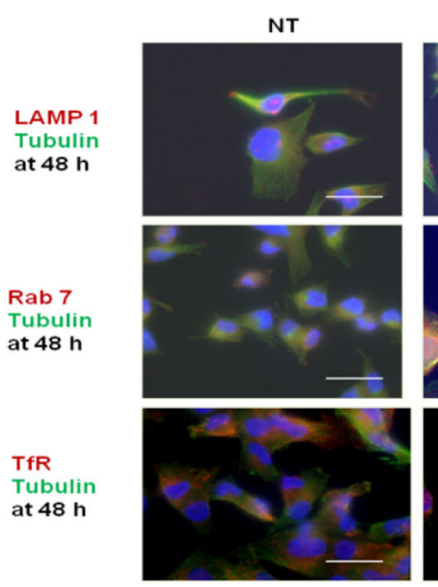

b

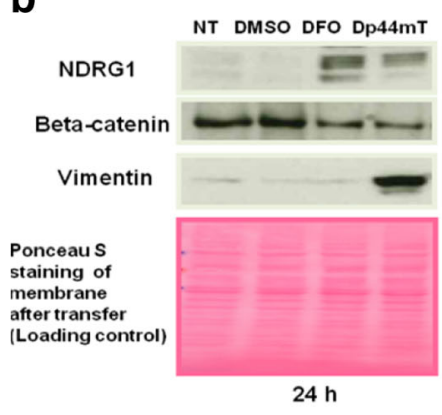

C

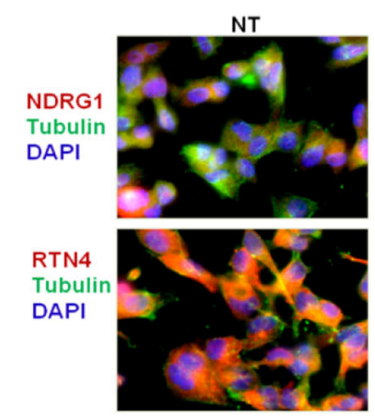

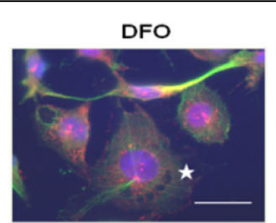
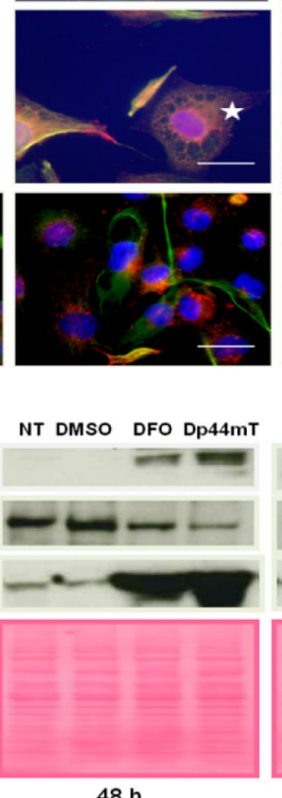

$48 \mathrm{~h}$
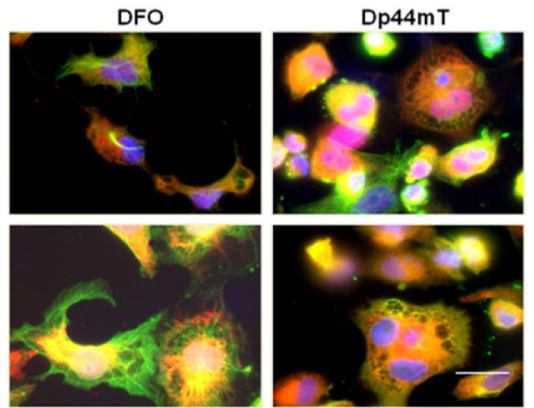

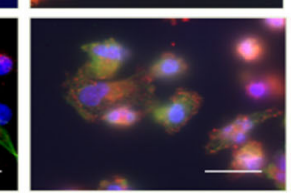

Dp44mt
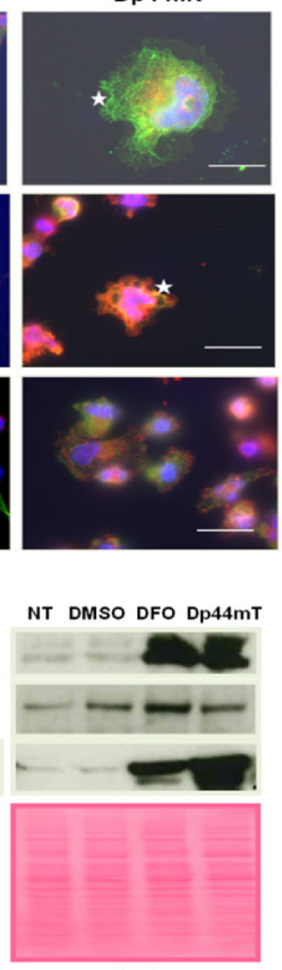

$72 \mathrm{~h}$

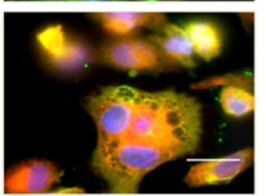

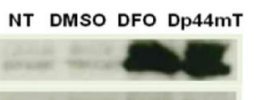

Fig. 4 Cell phenotype represents marked dilatation of the rough endoplasmic reticulum (ER). a Cells were treated with DFO or Dp44mT for 48 h, processed for immunofluorescence, and then observed under a fluorescence microscope. The vacuoles were identified to be positive for late endosome/macropinosome markers RAB7 and LAMP1. Stars indicate the localisation of RAB7 and LAMP1 surface proteins in the membranes of vacuoles. TfR was used as a marker for endocytosis. $\mathbf{b}$ Western blotting analysis of the expression of the epithelial to mesenchymal transition markers NDRG1, beta-catenin, and vimentin. The protein lysates were obtained from cells treated with DFO or Dp44mT for the times indicated. c Localisation of NDRG1 and RTN4 in vacuolated MDA-MB-231 cells. Cells were treated with DFO or Dp44mT for 48 h, processed for

immunofluorescence, and then observed under a confocal microscope. Cells were immunostained with NDRG1 and RTN4 (red) and anti-tubulin (green) antibodies and then analysed by fluorescence microscopy. Blue, DAPI. Scale bars: $100 \mu \mathrm{m}$

$72 \mathrm{~h}$ (Fig. 5b). SLIRP is required for the correct binding of mitochondrial mRNAs with the mitochondrial ribosome to enable efficient translation [69]; its decreased expression possibly indicates a down-regulation of mitochondrial gene expression.

\section{Iron chelation induces accumulation of fluid-filled vacuoles and LDs}

The same modifications in phenotype, cell behaviour, and some representative molecular patterns observed in MDA-
MB-231 were also found in the DFO- or Dp44mT-treated MDA-MB-157 breast cancer cell line (Fig. 6). This consistency in the results indicated the existence of a possible general correlation between iron depletion and the described effects. To further understand the observed modifications, we performed further determinations. As described earlier, after treatment with DFO or Dp44mT, large, swollen vacuoles appeared suddenly in MDA-MD-231 cells during the period from 48 to $72 \mathrm{~h}$, and the treated cells showed a massive accumulation of LDs that stained positively for Oil 


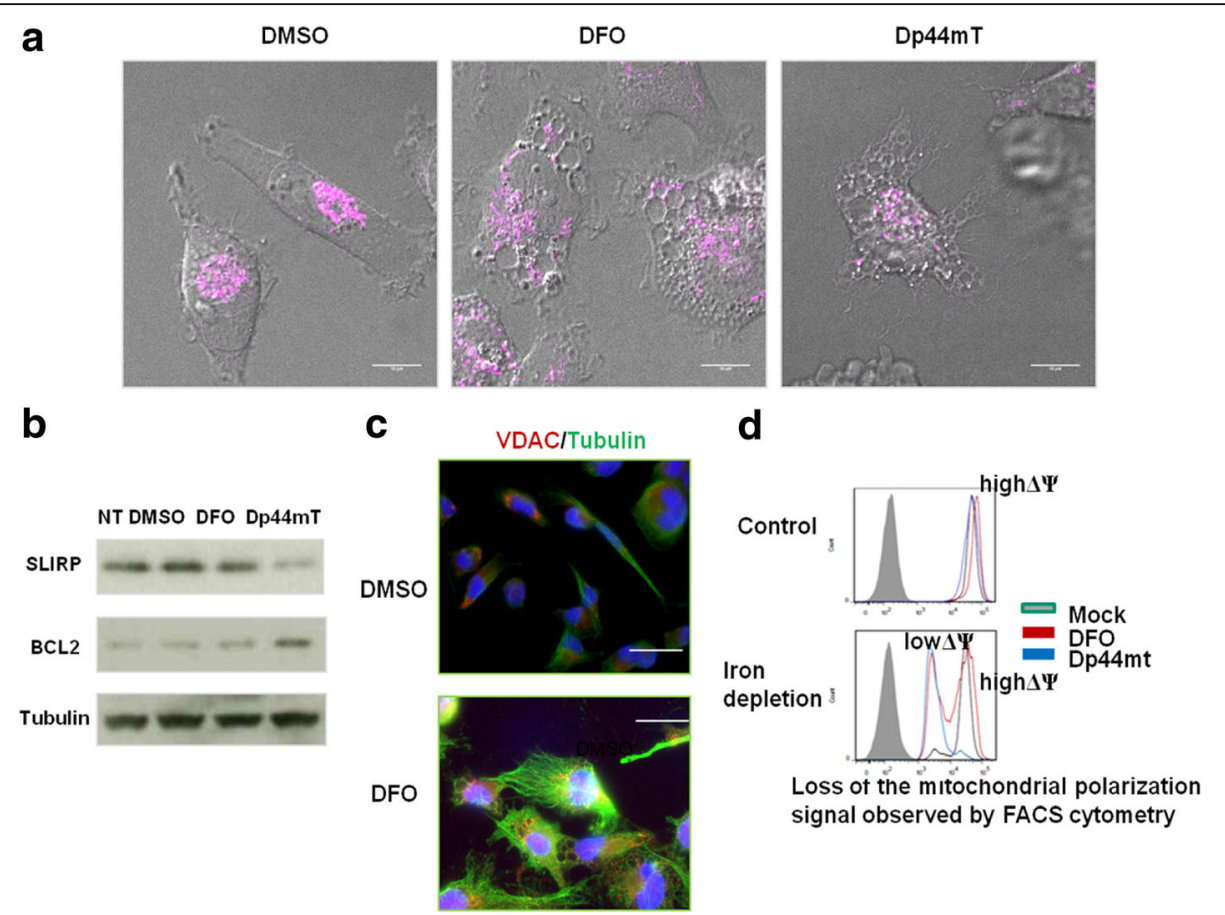

Fig. 5 Mitochondrial alterations in MDA-MB-231 cells induced by DFO or Dp44mT treatment. a Using confocal microscopy, alterations in mitochondrial morphology were evaluated using Rhodamine 123 (pink). Differential interference contrast (DIC) images and Rhodamine 123 fluorescence of control (left), DFO- (centre), and Dp44mT-treated cells (right). Only representative merged images are shown. The treatment with $100 \mu \mathrm{M}$ DFO or $5 \mu \mathrm{M}$ Dp44mT causes ER expansion with vacuolation, which is accompanied by breakage of the mitochondrial network. $\mathbf{b}$ Western blot analysis of SLIRP and BCL-2 expression in total lysates from untreated (NT or DMSO) and treated (DFO or Dp44mT) MDA-MB-231 cells. Tubulin was used as a loading control. c Cells were observed under an Olympus Fluo View 1000 fluorescence microscope. MDA-MB-231 cells, untreated or treated with DFO, were labelled with an anti-VDAC antibody (red) followed by appropriate secondary antibodies, anti-tubulin (green), and DAPI (blue). The cells were observed under an Olympus Fluo View 1000 confocal microscope. Only representative merged images are shown. Scale bars: 100 um. d Fluorescence-activated cell sorting (FACS) cytometry assessing mitochondrial depolarisation induced by DFO and Dp44mT

Red $\mathrm{O}$ in living as well as dying cells (Fig. 7). The LDs could be seen interspersed between the clear vacuoles. The treated cells exhibited considerably increased numbers of LDs, which is in agreement with the increased expression of PLIN2/ PLIN3 (Additional files 4, 6).

Non-linear light microscopy techniques, such as coherent anti-stokes Raman scattering (CARS) microscopy, was used to determine the vacuolar content and to visualise LDs within a single living cell [39]. The results showed that cells incorporate large amounts of extracellular material that accumulate into large and transparent vacuoles. Therefore, the expansion of the ER lumen to form vacuoles is possibly due to the scavenging of extracellular macromolecules that are needed to sustain cell survival in a nutrient-depleted environment. The swollen ER network accumulates lipids to form ER-derived LDs generated by budding mechanisms.

Finally, when cell swelling occurred $72 \mathrm{~h}$ after treatment with the chelators, vacuoles largely disappeared, and droplets were extruded by the cells. Many of these droplets converged to form branched aggregated of LDs (Fig. 7).
LDs are the intracellular sites for neutral lipid storage with total FA composition that can vary notably. The GCMS analysis of LDs derived from both DFO- and Dp44mT-treated cells showed that the major FAs present were oleic acid (29.3 and $38.4 \%$, respectively), stearic acid (27 and 31.5\%, respectively), arachidonic acid (ARA, 9.9 and 5.1\%, respectively), and palmitic acid (5.9 and 6.26\%, respectively) (Fig. 8). Notably, ARA can be metabolised into important lipid mediators and signalling molecules. Myristic acid is a saturated FA produced by FASN and was observed to be down-regulated by the treatments, representing only 0.56 and $0.88 \%$, respectively. Oleic acid, the most abundant FA among the analysed LDs, is a monounsaturated FA synthesised by the introduction of a cis double bond at the $\Delta-9$ position of stearic acid (C18) [70]. This oxidative conversion of stearic acid to oleic acid is catalysed by the NADH-dependent stearoyl-CoA desaturase (SCD), which was not observed to be differentially expressed in our proteomics analysis (Additional files 3, 4, 5,6 ), and that possibly explains the reduced SCD activity in hypoxia. The lipogenic acetyl-CoA carboxylase (ACACA) was also down-regulated (Additional files 3, 5). 
a

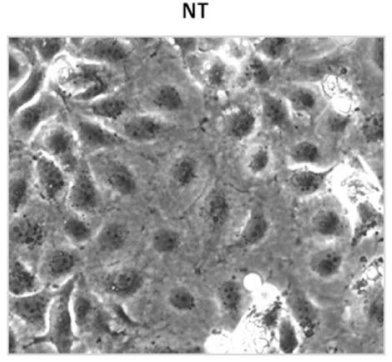

b
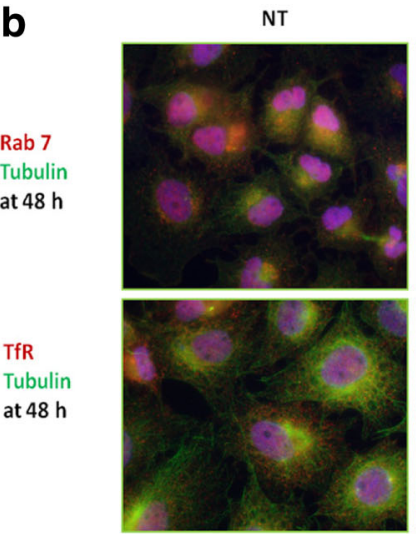
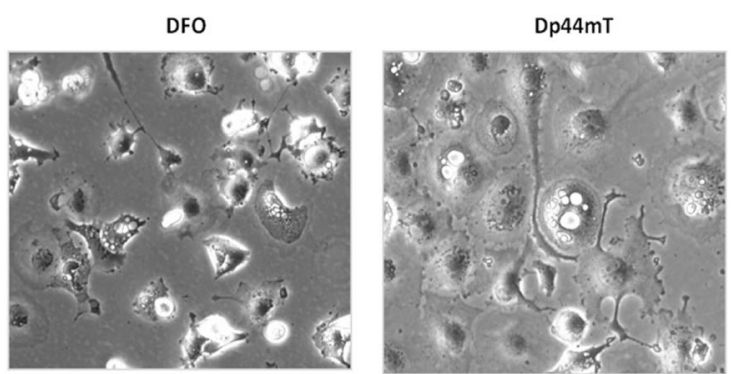

DFO

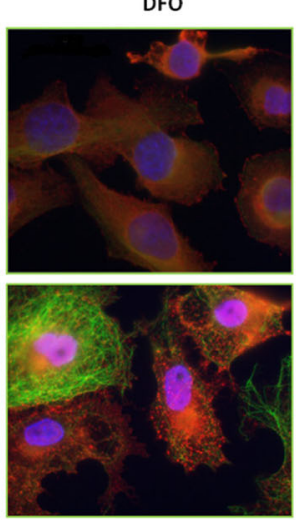

C

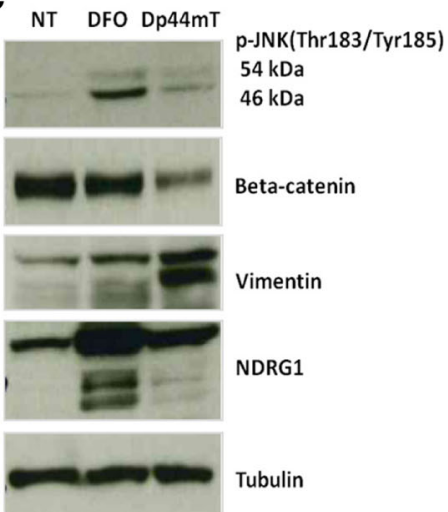

Fig. 6 Modifications of morphology and protein expression observed in the cell line MDA-MB-157 during iron depletion. a MDA-MB-157 cells were treated with $100 \mu \mathrm{M}$ of the iron chelator DFO for $72 \mathrm{~h}$ and photographed under an optical microscope. Scale bars: $100 \mu \mathrm{m}$. b MDA-MB-157 cells, untreated or treated with $100 \mu \mathrm{M}$ DFO for $48 \mathrm{~h}$, were labelled with anti-Rab 7 or anti-TfR antibody (red) followed by appropriate secondary antibodies, anti-tubulin (green), and DAPI (blue) and observed under an Olympus Fluo View 1000 fluorescence microscope. Only representative merged images are shown. Scale bars: $100 \mu \mathrm{m}$. c Western blot analyses of DFO- or Dp44mT-treated cells showing increased levels of phosphorylated JNK, beta-catenin, vimentin, and NDGR1. Tubulin was used as a loading control

Previous studies have demonstrated that reduced SCD activity in hypoxic cells was associated with the scavenging of exogenous monounsaturated FAs to maintain a viable desaturation index [71]. Otherwise, a sustained ER stress was observed. In contrast, we observed that the majority of FAs in LDs were unsaturated; the desaturation index was very high (34\% saturated vs $66 \%$ desaturated for DFO-treated cells and 39\% saturated vs $61 \%$ desaturated for Dp44mT-treated cells), and oleic acid (29.3 and 38.4\%, respectively) was more abundant than stearic acid (27 and $31.5 \%$, respectively).

The role of LDs was not investigated in the present study; our results reveal a link between iron depletion, reduced mitochondrial activity, and LD accumulation and show that the mono-saturated oleic acid is the most abundant FA present in the LDs.

\section{Discussion}

Iron is an essential nutrient required for many biosynthetic and metabolic processes, such as haem and sulphur-iron cluster biosynthesis, oxygen transport, mitochondrial respiration, lipid desaturation, and DNA replication [72]. Compared with normal cells, cancer cells require a large amount of iron for several essential processes. Iron bio- availability can be affected by the lack of functional vasculature in tumours, resulting in a hypoxic/anaemic tumour microenvironment. Iron affects the HIF pathway, and iron deficiency can produce responses similar to hypoxia, as observed from the effects of iron chelation in cell culture and in humans [73]. However, the effects of iron deficiency on hypoxia behaviour and signalling mechanisms have not been completely understood.

Our study provides novel insights on how iron deprivation modulates the cell structure and the proteomic and metabolic pathways in breast cancer cells. Prolonged iron deficiency in cultured tumour cells induces significant changes in cellular phenotypes and mesenchymal markers and an adaptive cellular response similar to that induced by hypoxic stress, prior to killing the tumour cells [74]. HIF-1 was up-regulated in MDA-MB-231 cells (Additional files 4, 6) after treatment with iron chelators, sustaining EMT and hypoxia programs; mesenchymal marker genes, NDRG1 and vimentin, increased in parallel with the decrease in Ecadherin expression and the increase in CD44 and CD166 expression, which are well-known cancer stem cell markers [75] (Additional files 4, 6). Remodelling of iron-starved cells showed a marked reduction in translation and the increase of redox pathways, along with perinuclear mitochondrial 


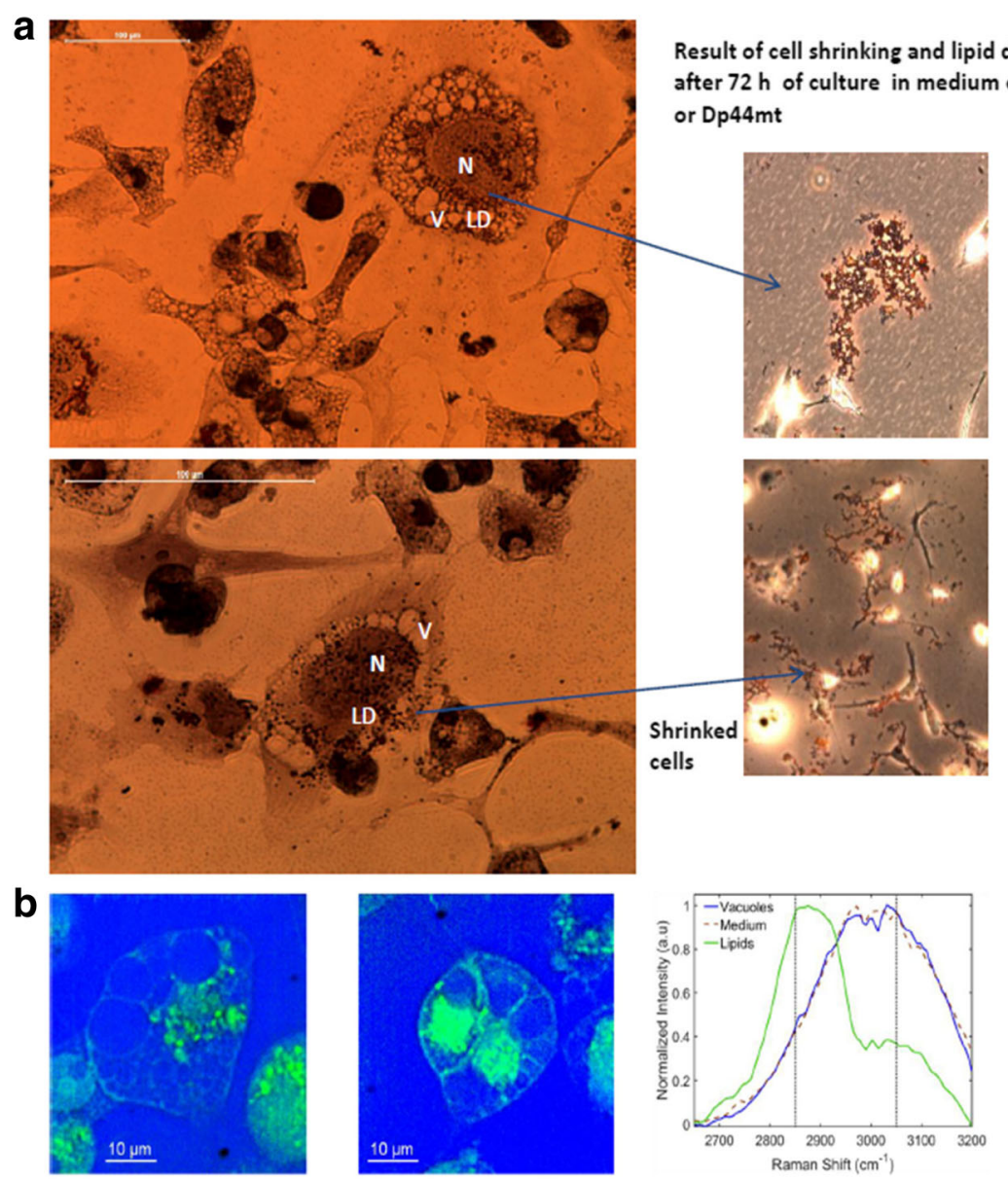

Fig. 7 Oil Red O staining and label-free CARS imaging of MDA-MB-231 cells after DFO treatment. a Oil Red O staining of MDA-MB-231 cells treated with DFO for $48 \mathrm{~h}$. Images on the left show cells under a microscope at magnifications of 200x and 400x. Cells were stained with Oil Red O and counterstained with haematoxylin to show nuclei $(N)$. The cells adopted a round morphology and accumulated large cytoplasmic vacuoles $(V)$ in which accumulation of lipids (LD) was interspersed. Images on the right show examples of how cell morphology evolves after $72 \mathrm{~h}$ of treatment. Cells shrink before dying with a consistent release of aggregates of floating lipid droplets. $\mathbf{b}$ CARS composite images of MDA-MB-231 cells treated with $100 \mu \mathrm{M}$ DFO for $72 \mathrm{~h}$. Panels show the images of lipids in green $\left(2850 \mathrm{~cm}^{-1}\right)$ and of the vacuoles and medium $\left(3050 \mathrm{~cm}^{-1}\right)$; CARS spectra are also reported

clustering disruption, depolarisation, and a hypoxia-like reprogramming of metabolism. The reprogramming suggests an adaptation of the cancer cells to iron deprivation, which is accompanied by the augmentation of mesenchymal characteristics.

The expanded ER formed large vacuoles and a complex network of membranes around the nucleus. Coalescent spherical units of translucent material around the nucleus were stained with lipid dyes such as Oil Red O and observed using Raman spectroscopy. These structural modifications occurred in parallel with the increased expression of ER markers (NDRG1 and RTN4) and LD-associated proteins (PLIN2/PLIN3). Using Raman spectroscopy, we could also determine that the vacuoles contained sacs of fluid-filled extracellular medium. These modifications increased with time of treatment with DFO or Dp44mT and were tolerated by the cells before they underwent cytoplasmic collapse, succumbing to a non-apoptotic and non-autophagic type of death.

Thus, under iron deprivation, breast cancer cells exhibited a large amount of fluid-filled vacuoles and lipid accumulation. A type of extensive vacuolation followed by cell death, known as methuosis, or death by macropinocytosis has been previously described as an efficient method for inducing cell death in different types of cancers [76, 77]. Proteomics analyses of pre-death cells clearly indicated a striking metabolic plasticity, based on the scavenging of nutrients destined for short-term cell survival.

By recycling nutrients from intracellular macromolecules, autophagy represents an important cellular strategy to sustain viability during periods of limited nutrient availability [78]. We observed a blocking of autophagy and possible loss of cell viability, which may be derived from the cytoplasmic hyper-vacuolisation associated 

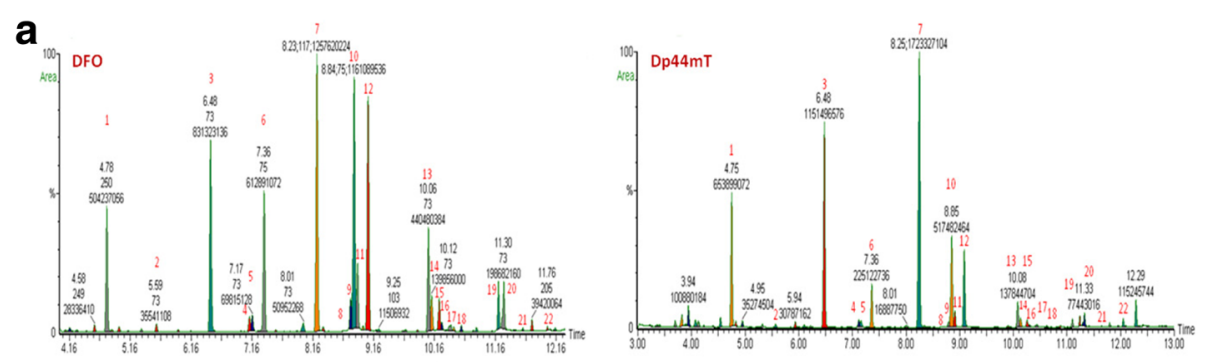

b

$\begin{array}{cc}\text { Peak } & \text { Fatty acid } \\ 2 & \text { C14:0 } \\ 4 & \text { C16:1A } \\ 5 & \text { C16:1B } \\ 6 & \text { C16:0 } \\ 8 & \text { C18:3 } \\ 9 & \text { C18:2 } \\ 10 & \text { C18:1A } \\ 11 & \text { C18:1B } \\ 12 & \text { C18:0 } \\ 13 & \text { C20:4 } \\ 14 & \text { C20:5 } \\ 15 & \text { C20:3 } \\ 16 & \text { C20:2 } \\ 17 & \text { C20:1 } \\ 18 & \text { C20:0 } \\ 19 & \text { C22:6 } \\ 20 & \text { C22:5 } \\ 21 & \text { C22:0 } \\ \text { IS } & 4 \text {-pentylbenzoic acid (1);C15 } \\ & \text { (3);C17:0(7);C23:0(22) }\end{array}$

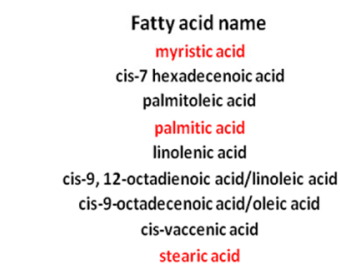

cis-5, 8, 11, 14-eicosatetraenoic acid/arachidonic acid

14 , cis-5, 8, 11, 14, 17-eicosapentaenoic acid

cis-8, 11, 14-eicosatrienoic acid

cis-11, 14-eicosadienoic acid

cis-11-eicosenoic acid/gondoic acid arachidic acid

cis-4, 7, 10, 13, 16, 19-docosahexaenoic acid

cis-7,10,13,16,19-docosapentaenoic acid behenic acid

C

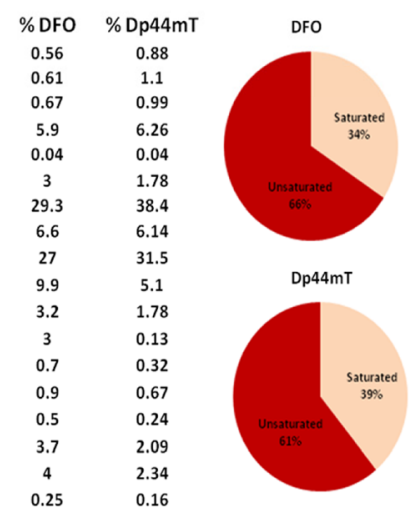

Fig. 8 FA quantification by GC-MS. a GC-MS analysis of FAs obtained from LDs. FAs were extracted from LDs purified from DFO- and Dp44mTtreated cells and subjected to GC-MS analysis as described under 'Materials and Methods'. The major FA subtypes in the LDs were oleic, stearic, and arachidonic acids. b Peak number, name of the FA, and FA quantification reported as peak area percentage. $\mathbf{c}$ Proportions of unsaturated and saturated FAs

with the loss of metabolic capacity (as indicated by the decrease in mitochondrial membrane potential) and plasma membrane integrity, until osmotic pressure permitted. Cells formed non-acidic and non-autophagic vacuoles, with massive cytoplasmic vacuolations developing from the dilation of the ER lumen. The formation of intracellular vacuoles and subsequent cell death indicated that the events occurring in the ER initiate this destructive pathway. This massive dilation of the ER finally culminates in a paraptosis-like cell death [3], releasing LDs into the extracellular environment. Consequently, in vivo anaemic/hypoxic regions in tumours with poor iron availability may be considered to be at risk for dissemination of possible bioactive vesicles.

The association between LD metabolism and cancer cell survival and metabolism is unknown. LD accumulation probably results from the increased lipid scavenging activity in MDA-MB-231 cells, rather than augmented lipogenesis [79]. In accordance with this view, we observed a reduction in FASN levels, which could limit the initial step in FA biosynthesis, and the KEGG indication of an increase in lipid degradation, suggesting that cancer cells scavenge lipids from the extracellular environment. Importantly, hypoxic cells exhibit increased uptake of unsaturated lipids from their environment, thus bypassing the requirement for FA desaturation [80]. In fact, FA desaturases, mainly SCD, are among the most sensitive targets of oxygen and iron starvation [81]. We observed only a decrease in delta-6 desaturase (FADS2) abundance in treated cells accompanied by the increase in LD accumulation. Based on this result, we expected a change in lipid composition with an increase in saturated FAs. Instead, a large amount of monounsaturated oleic acid was observed in DFO-and Dp44mT-induced LDs (29.3 and $38.4 \%$ of total FAs), which is generally regarded as cytoprotective [82]. Although an abundance of saturated stearic acid (27 and $31.5 \%$ ), possibly due to the reduction of desaturase functions, was also observed in the LDs, the unsaturated/saturated FA ratio indicated a greater content of unsaturated FAs. Some polyunsaturated FAs observed in the LD analysis have distinct and contrasting effects in cancer: arachidonic acid mostly exhibits pro-tumourigenic effects [82]; eicosapentaenoic (EPA; 20:5, $\omega-3)$ and docosahexaenoic acids (DHA; 22:6, $\omega-3$ ) possess antitumourigenic, anti-inflammatory, and pro-apoptotic effects in cancer cells [83].

The mitochondrial dysfunction observed in our studies does not support an active metabolic role for LDs and further suggests that other compensatory metabolic adaptations to support cell survival under conditions of iron starvation must be considered. 


\section{Conclusions}

Iron depletion in breast cancer cells induced mitochondrial dysfunction and inhibited the activities of several essential enzymes requiring iron as a cofactor. The morphological, proteomic, and metabolic aspects of our study support the view that cells develop a short-term stress tolerance under iron depletion, activating a hypoxia-like program with the uptake of nutrients from the extracellular fluid-phase by macropinocytosis. However, the blockage of autophagy and the dysfunctional mitochondrial respiration do not permit a sufficient level of catabolism to sustain long-term cell survival. Drastic cell structure changes guided by ER expansion create two main storage compartments: extracellular fluidfilled vacuoles and LDs that accumulate lipids because of the loss of FA oxidation. LDs released after cell death have potential benefits for study as metabolic entities to understand the mechanistic and clinical aspects of tumour biology in future studies.

\section{Additional files} Additional file 1: Table S1. List of primary antibodies used for western
blot analysis. (DOCX $11 \mathrm{~kb}$ )

Additional file 2: FACS analysis of cell cycle. (PPTX 200 kb)

Additional files 3: Reports of statistical results, specifically up- and downregulated proteins in DFO- or Dp44mT-treated cells with respect to control. Down-regulated in DFO- treated cells versus untreated. (XLS 618 kb)

Additional file 4: Up-regulated in DFO-treated cells versus untreated. (XLS $375 \mathrm{~kb}$ )

Additional file 5: Down-regulated in Dp44mT-treated cells versus untreated. (XLS $721 \mathrm{~kb}$ )

Additional file 6: Up-regulated in Dp44mT-treated cells versus untreated. Differential expression was considered as significant if (1) a protein was present only in untreated or treated cells or (2) its normalised (according to the LFQ algorithm) intensity resulted in a statistical difference, as calculated by the welch's t-test (t-test cut-off at $p$ value $=0.0167$ ). These data have been deposited to the ProteomeXchange Consortium (http://proteomecentral.proteomexchange.org/cgi/GetDataset) via the PRIDE (Vizcaíno et al., 2016 PubMed ID: 26527722) partner repository with the dataset identifier PXD007595. (XLS 537 kb)

Additional file 7: Ingenuity Pathway analysis revealing the pathways significantly changed after the DFO/Dp44mT treatments. (PPTX 397 kb)

\section{Abbreviations}

(PLIN) 2: Perilipin; ACACA: lipogenic acetyl-CoA carboxylase; ANOVA: Analysis of variance; ARA: Arachidonic acid; ATCC: American Type Culture Collection; BCA: Bicinchoninic acid; BCL-2: B-cell lymphoma 2; BHT: Butylated hydroxy toluene; CARS: Coherent anti-Stokes Raman scattering; DAPI: 4',6-diamidino-2phenylindole; DAPI: 4',6-diamidino-2-phenylindole; differential interference contrast; DEGS: D4-dihydroceramide (DhCer) desaturase; DFO: Deferoxamine; DHA: Docosahexaenoic acids; DMEM: Dulbecco's modified Eagle's medium; DMSO: Dimethyl sulfoxide; Dp44mT: Di-2-pyridylketone 4,4-dimethyl-3thiosemicarbazone; DpT: Di-2-pyridylketone thiosemicarbazone; EMT: Epithelialmesenchymal transition; EPA: Eicosapentaenoic; ER: Endoplasmic reticulum; FA: Fatty acid; FACS: Fluorescence-activated cell sorting; FADS: FA desaturase; FADS2: delta-6 desaturase; FASN: FA synthase; FBS: Foetal bovine serum; FDR: False discovery rate; Fru-6P: Fructose-6-phosphate; GC-MS: Gas chromatography-mass spectrometry; GFPT1: Gln-fructose-6-phosphate transaminase 1; GO: Gene ontology; GOT: Glutamic-oxaloacetic transaminase; HBP: Hexosamine biosynthetic pathway; HIF: Hypoxia-inducible factor; HMGB1: High mobility group box 1; IF: Immunofluorescence; KEGG: Kyoto Encyclopedia of Genes and Genomes; LAMP 1: Lysosomal-associated
}

membrane protein; LC3: microtubule-associated protein light chain 3 (LC3); LD: Lipid droplets; LQF: Label-free quantification; LY: Lucifer yellow; LysoTracker: Lysosomotropic metachromatic fluorochrome; MCRALS: Multivariate Curve Resolution Alternating Least Squares; MTT: 3-[4,5dimethylthiazol-2-yl]-2,5-diphenyltetrazolium bromide; thiazolyl blue; NDRG1: Nmyc downstream-regulated gene 1; PARP: Poly (ADP-ribose) polymerase-1; ROS: Reactive oxygen species; RTN4: Reticulon 4; SCD: Stearoyl-CoA desaturase; SEM: Standard error of the mean; SLC1A5: Sodium-dependent neutral amino acid transporter type 2; SLIRP: SRA stem-loop interacting RNA-binding protein; STRING: Search Tool for the Retrieval of INteracting Genes/proteins; TCA: Tricarboxylic acid; TfR: Transferrin receptor

\section{Acknowledgements}

This study was supported by the IG_13208 grant from Associazione italiana per la ricerca sul cancro (AIRC), the Italian Ministry of Public Health and the European Research Council (ERC) under the European Union's Horizon 2020 research and innovation programme (grant agreement No 648615). We thank the PRIDE team. The authors also acknowledge the help of Gabriella Abolafio for flow cytometry and Maria Teresa Radice for technical assistance at Fondazione IRCCS Istituto Nazionale dei Tumori, Milan.

\section{Availability of data and materials}

The mass spectrometry proteomics data have been deposited to the ProteomeXchange Consortium (http://proteomecentral.proteomexchange.org/ cgi/GetDataset) via the PRIDE (Vizcaíno et al., 2016 PubMed ID: 26527722) partner repository with the dataset identifier PXD007595.

\section{Authors' contributions}

Conceptualization: IB and GT; Methodology: MD, ET, PC; Investigation: IB, EM, CC, FC and VK; Resources: IB, GT, CC and DP; Data curation, IB, GT, CC and DP; Writing original draft: IB; Visualization: IB, GT, FC, VK, and CC; Supervision: $\mathrm{DP}$ and GT. All authors read and approved the final manuscript.

\section{Ethics approval and consent to participate}

Not applicable.

\section{Consent for publication}

All authors consented to the publication of this article.

\section{Competing interests}

The authors declare that they have no competing interests.

\section{Publisher's Note}

Springer Nature remains neutral with regard to jurisdictional claims in published maps and institutional affiliations.

\section{Author details}

${ }^{1}$ Fondazione IRCCS Istituto Nazionale dei Tumori, via G. Amadeo 42, Milan 20133, Italy. ${ }^{2}$ Dipartimento di Medicina Veterinaria (DiMeVet), University of Milan, Milan I-20133, Italy. ${ }^{3}$ Molecular Targeting Unit, Fondazione IRCCS Istituto Nazionale dei Tumori, Via Amadeo 42, 20133 Milan, Italy. ${ }^{4}$ IFN-CNR, Dipartimento di Fisica, Politecnico di Milano, Piazza Leonardo da Vinci 32, 20133 Milan, Italy. ${ }^{5}$ Laboratory of Clinical Pathology and Medical Genetics, Fondazione IRCCS 'Carlo Besta' Istituto Neurologico, Via Amadeo 42, 20133 Milan, Italy. ${ }^{6}$ Center for Nano Science and Technology at Polimi, Istituto Italiano di Tecnologia, 20133 Milan, Italy. 'Fondazione Filarete, I-20139 Milan, Italy.

Received: 9 November 2017 Accepted: 15 March 2018 Published online: 03 April 2018

\section{References}

1. Lieu PT, Heiskala M, Peterson PA, Yang Y. The roles of iron in health and disease. Mol Asp Med. 2001;22(1-2):1-87.

2. Whitnall M, Howard J, Ponka P, Richardson DR. A class of iron chelators with a wide spectrum of potent antitumor activity that overcomes resistance to chemotherapeutics. Proc Natl Acad Sci U S A. 2006;103(40):14901-6.

3. Lee D, Kim IY, Saha S, Choi KS. Paraptosis in the anti-cancer arsenal of natural products. Pharmacol Ther. 2016;162:120-33.

4. Buss JL, Greene BT, Turner J, Torti FM, Torti SV. Iron chelators in cancer chemotherapy. Curr Top Med Chem. 2004;4(15):1623-35. 
5. Kalinowski DS, Richardson DR. The evolution of iron chelators for the treatment of iron overload disease and cancer. Pharmacol Rev. 2005;57(4):547-83.

6. Huang X. Does iron have a role in breast cancer? Lancet Oncol. 2008;9(8): 803-7.

7. Heath JL, Weiss JM, Lavau CP, Wechsler DS. Iron deprivation in cancerpotential therapeutic implications. Nutrients. 2013:5(8):2836-59.

8. Olivieri NF, Brittenham GM. Iron-chelating therapy and the treatment of thalassemia. Blood. 1997;89(3):739-61.

9. Schelman WR, Morgan-Meadows S, Marnocha R, Lee F, Eickhoff J, Huang W, et al. A phase I study of Triapine in combination with doxorubicin in patients with advanced solid tumors. Cancer Chemother Pharmacol. 2009; 63(6):1147-56.

10. Borgna-Pignatti C, Rugolotto S, De Stefano P, Zhao H, Cappellini MD, Del Vecchio GC, et al. Survival and complications in patients with thalassemia major treated with transfusion and deferoxamine. Haematologica. 2004; 89(10):1187-93.

11. Moon JH, Jeong JK, Park SY. Deferoxamine inhibits TRAlL-mediated apoptosis via regulation of autophagy in human colon cancer cells. Oncol Rep. 2015;33(3):1171-6.

12. Brard L, Granai $\mathrm{CO}$, Swamy N. Iron chelators deferoxamine and diethylenetriamine pentaacetic acid induce apoptosis in ovarian carcinoma. Gynecol Oncol. 2006;100(1):116-27.

13. Hann HW, Stahlhut MW, Rubin R, Maddrey WC. Antitumor effect of deferoxamine on human hepatocellular carcinoma growing in athymic nude mice. Cancer. 1992;70(8):2051-6.

14. Kalinowski DS, Sharpe PC, Bernhardt PV, Richardson DR. Design, synthesis, and characterization of new iron chelators with anti-proliferative activity: structure-activity relationships of novel thiohydrazone analogues. J Med Chem. 2007;50(24):6212-25.

15. Chen Z, Zhang D, Yue F, Zheng M, Kovacevic Z, Richardson DR. The iron chelators Dp44mT and DFO inhibit TGF-beta-induced epithelialmesenchymal transition via up-regulation of N-Myc downstream-regulated gene 1 (NDRG1). J Biol Chem. 2012;287(21):17016-28.

16. Dixon KM, Lui GY, Kovacevic Z, Zhang D, Yao M, Chen Z, et al. Dp44mT targets the AKT, TGF-beta and ERK pathways via the metastasis suppressor NDRG1 in normal prostate epithelial cells and prostate cancer cells. Br J Cancer. 2013;108(2):409-19.

17. Kovacevic Z, Chikhani S, Lui GY, Sivagurunathan S, Richardson DR. The ironregulated metastasis suppressor NDRG1 targets NEDD4L, PTEN, and SMAD4 and inhibits the PI3K and Ras signaling pathways. Antioxid Redox Signal. 2013;18(8):874-87.

18. Sun J, Zhang D, Zheng Y, Zhao Q, Zheng M, Kovacevic Z, et al. Targeting the metastasis suppressor, NDRG1, using novel iron chelators: regulation of stress fiber-mediated tumor cell migration via modulation of the ROCK1/ pMLC2 signaling pathway. Mol Pharmacol. 2013;83(2):454-69.

19. Liu W, Xing F, liizumi-Gairani M, Okuda H, Watabe M, Pai SK, et al. N-myc downstream regulated gene 1 modulates Wnt-beta-catenin signalling and pleiotropically suppresses metastasis. EMBO Mol Med. 2012;4(2):93-108.

20. Liu W, Yue F, Zheng M, Merlot A, Bae DH, Huang M, et al. The protooncogene c-Src and its downstream signaling pathways are inhibited by the metastasis suppressor, NDRG1. Oncotarget. 2015:6(11):8851-74.

21. Page EL, Chan DA, Giaccia AJ, Levine M, Richard DE. Hypoxia-inducible factor-1alpha stabilization in nonhypoxic conditions: role of oxidation and intracellular ascorbate depletion. Mol Biol Cell. 2008;19(1):86-94.

22. Xie $\mathrm{H}$, Simon MC. Oxygen availability and metabolic reprogramming in cancer. J Biol Chem. 2017;292(41):16825-32.

23. Bisaro B, Mandili G, Poli A, Piolatto A, Papa V, Novelli F, et al. Proteomic analysis of extracellular vesicles from medullospheres reveals a role for iron in the cancer progression of medulloblastoma. Mol cell Ther. 2015;3:8. 0150045-3. eCollection 2015

24. Simcox JA, McClain DA. Iron and diabetes risk. Cell Metab. 2013;17(3):329-41.

25. Caccia D, Micciche F, Cassinelli G, Mondellini P, Casalini P, Bongarzone I. Dasatinib reduces FAK phosphorylation increasing the effects of RPI-1 inhibition in a RET/ PTC1-expressing cell line. Mol Cancer. 2010;9:278. 4598-9-278

26. Gorla L, Mondellini P, Cuccuru G, Micciche F, Cassinelli G, Cremona M, et al. Proteomics study of medullary thyroid carcinomas expressing RET germ-line mutations: identification of new signaling elements. Mol Carcinog. 2009; 48(3):220-31.

27. Bozzi F, Mogavero A, Varinelli L, Belfiore A, Manenti G, Caccia C, et al. MIF/ CD74 axis is a target for novel therapies in colon carcinomatosis. J Exp Clin Cancer Res. 2017;36(1):16. 016-0475-z
28. Dell'Orco M, Milani P, Arrigoni L, Pansarasa O, Sardone V, Maffioli E, et al. Hydrogen peroxide-mediated induction of SOD1 gene transcription is independent from Nrf2 in a cellular model of neurodegeneration. Biochim Biophys Acta. 2016;1859(2):315-23.

29. Coccetti P, Tripodi F, Tedeschi G, Nonnis S, Marin O, Fantinato S, et al. The CK2 phosphorylation of catalytic domain of $\mathrm{Cdc} 34$ modulates its activity at the G1 to $S$ transition in Saccharomyces cerevisiae. Cell Cycle. 2008;7(10):1391-401.

30. Cox J, Mann M. MaxQuant enables high peptide identification rates, individualized p.P.B.-range mass accuracies and proteome-wide protein quantification. Nat Biotechnol. 2008;26(12):1367-72.

31. Zanotti L, Angioni R, Cali B, Soldani C, Ploia C, Moalli F, et al. Mouse mesenchymal stem cells inhibit high endothelial cell activation and lymphocyte homing to lymph nodes by releasing TIMP-1. Leukemia. 2016;30(5):1143-54.

32. Vizcaino JA, Csordas A, del-Toro N, Dianes JA, Griss J, Lavidas I, et al. 2016 update of the PRIDE database and its related tools. Nucleic Acids Res. 2016; 44(D1):D447-56

33. Szklarczyk D, Morris JH, Cook H, Kuhn M, Wyder S, Simonovic M, et al. The STRING database in 2017: quality-controlled protein-protein association networks, made broadly accessible. Nucleic Acids Res. 2017:45(D1):D362-8.

34. Kanehisa M, Furumichi M, Tanabe M, Sato Y, Morishima K. KEGG: new perspectives on genomes, pathways, diseases and drugs. Nucleic Acids Res. 2017;45(D1):D353-61.

35. Kanehisa M, Goto S. KEGG: Kyoto encyclopedia of genes and genomes. Nucleic Acids Res. 2000;28(1):27-30.

36. Kanehisa M, Sato Y, Kawashima M, Furumichi M, Tanabe M. KEGG as a reference resource for gene and protein annotation. Nucleic Acids Res. 2016;44(D1):D457-62.

37. Sato S, Fukasawa M, Yamakawa Y, Natsume T, Suzuki T, Shoji I, et al. Proteomic profiling of lipid droplet proteins in hepatoma cell lines expressing hepatitis C virus core protein. J Biochem. 2006;139(5):921-30.

38. Terao J, Magarian RA, Brueggemann G, King MM. Methods of extraction and high-performance liquid chromatographic analysis of butylated hydroxytoluene from the tissues and serum of rats. Anal Biochem. 1985; 151(2):445-54.

39. Crisafi F, Kumar V, Scopigno T, Marangoni M, Cerullo G, Polli D. In-line balanced detection stimulated Raman scattering microscopy. Sci Rep. 2017: 7(1):10745. 017-09839-1

40. Tauler R. Multivariate curve resolution applied to second order data. J Chemom. 1995;9:31-58. https://doi.org/10.1016/0169-7439(95)00047-X.

41. de Juan A, Tauler R. Chemometrics applied to unravel multicomponent processes and mixtures: Revisiting latest trends in multivariate resolution. Anal Chim Acta. 2003;500:195-210. https:/doi.org/10.1016/S0003-2670(03)00724-4.

42. DeBerardinis RJ, Chandel NS. Fundamentals of cancer metabolism. Sci Adv. 2016;2(5):e1600200.

43. Nose F, Yamaguchi T, Kato R, Aiuchi T, Obama T, Hara S, et al. Crucial role of perilipin-3 (TIP47) in formation of lipid droplets and PGE2 production in $\mathrm{HL}$ 60-derived neutrophils. PLoS One. 2013;8(8):e71542.

44. Vaittinen M, Walle P, Kuosmanen E, Mannisto V, Kakela P, Agren J, et al. FADS2 genotype regulates delta-6 desaturase activity and inflammation in human adipose tissue. J Lipid Res. 2016;57(1):56-65.

45. Geeraert L, Mannaerts GP, van Veldhoven PP. Conversion of dihydroceramide into ceramide: involvement of a desaturase. Biochem J. 1997;327(Pt 1):125-32.

46. Shaul YD, Freinkman E, Comb WC, Cantor JR, Tam WL, Thiru P, et al, Dihydropyrimidine accumulation is required for the epithelial-mesenchymal transition. Cell. 2014:158(5):1094-109.

47. Guillaumond F, Leca J, Olivares O, Lavaut MN, Vidal N, Berthezene P, et al. Strengthened glycolysis under hypoxia supports tumor symbiosis and hexosamine biosynthesis in pancreatic adenocarcinoma. Proc Natl Acad Sci U S A. 2013;110(10):3919-24.

48. Caplan S, Hartnell LM, Aguilar RC, Naslavsky N, Bonifacino JS. Human Vam6p promotes lysosome clustering and fusion in vivo. J Cell Biol. 2001;154(1):109-22.

49. Martinet W, Knaapen MW, Kockx MM, De Meyer GR. Autophagy in cardiovascular disease. Trends Mol Med. 2007;13(11):482-91.

50. Chen PM, Gombart ZJ, Chen JW. Chloroquine treatment of ARPE-19 cells leads to lysosome dilation and intracellular lipid accumulation: possible implications of lysosomal dysfunction in macular degeneration. Cell Biosci. 2011;1(1):10. 3701-1-10

51. Ling D, Salvaterra PM. Brain aging and Abeta(1)(-)(4)(2) neurotoxicity converge via deterioration in autophagy-lysosomal system: a conditional Drosophila model linking Alzheimer's neurodegeneration with aging. Acta Neuropathol. 2011;121(2):183-91. 
52. Juopperi TA, Kim WR, Chiang CH, Yu H, Margolis RL, Ross CA, et al. Astrocytes generated from patient induced pluripotent stem cells recapitulate features of Huntington's disease patient cells. Mol Brain. 2012;5: 17. 6606-5-17

53. Barth S, Glick D, Macleod KF. Autophagy: assays and artifacts. J Pathol. 2010; 221(2):117-24.

54. Klionsky DJ. Developing a set of guidelines for your research field: a practical approach. Mol Biol Cell. 2016;27(5):733-8.

55. Phadwal K, Watson AS, Simon AK. Tightrope act: autophagy in stem cell renewal, differentiation, proliferation, and aging. Cell Mol Life Sci. 2013;70(1):89-103.

56. Lamb CA, Yoshimori T, Tooze SA. The autophagosome: origins unknown, biogenesis complex. Nat Rev Mol Cell Biol. 2013;14(12):759-74.

57. Pierzynska-Mach A, Janowski PA, Dobrucki JW. Evaluation of acridine orange, LysoTracker red, and quinacrine as fluorescent probes for long-term tracking of acidic vesicles. Cytometry A. 2014;85(8):729-37.

58. Belichenko PV, Dahlstrom A. Dual channel confocal laser scanning microscopy of lucifer yellow-microinjected human brain cells combined with Texas red immunofluorescence. J Neurosci Methods. 1994;52(2):111-8.

59. Yuan J, Lipinski M, Degterev A. Diversity in the mechanisms of neuronal cell death. Neuron. 2003;40(2):401-13.

60. Sahni S, Bae DH, Lane DJ, Kovacevic Z, Kalinowski DS, Jansson PJ, et al. The metastasis suppressor, N-myc downstream-regulated gene 1 (NDRG1), inhibits stress-induced autophagy in cancer cells. J Biol Chem. 2014;289(14): 9692-709.

61. Ureshino H, Murakami $Y$, Watari $K$, Izumi H, Kawahara A, Kage M, et al. Nmyc downstream regulated gene 1 (NDRG1) promotes metastasis of human scirrhous gastric cancer cells through epithelial mesenchymal transition. PLoS One. 2012;7(7):e41312.

62. Kovacevic Z, Richardson DR. The metastasis suppressor, Ndrg-1: a new ally in the fight against cancer. Carcinogenesis. 2006;27(12):2355-66.

63. Kalluri R, Weinberg RA. The basics of epithelial-mesenchymal transition. J Clin Invest. 2009;119(6):1420-8.

64. Nixon-Abell J, Obara CJ, Weigel AV, Li D, Legant WR, Xu CS, et al. Increased spatiotemporal resolution reveals highly dynamic dense tubular matrices in the peripheral ER. Science. 2016;354(6311):aaf3928. Epub 2016 Oct 27

65. Brasaemle DL. Thematic review series: adipocyte biology. The perilipin family of structural lipid droplet proteins: stabilization of lipid droplets and control of lipolysis. J Lipid Res. 2007:48(12):2547-59.

66. Wolins NE, Rubin B, Brasaemle DL. TIP47 associates with lipid droplets. J Biol Chem. 2001:276(7):5101-8

67. Lopez-Crisosto C, Pennanen C, Vasquez-Trincado C, Morales PE, Bravo-Sagua $R$, Quest AFG, et al. Sarcoplasmic reticulum-mitochondria communication in cardiovascular pathophysiology. Nat Rev Cardiol. 2017;14(6):342-60.

68. Galloway CA, Yoon Y. Mitochondrial morphology in metabolic diseases. Antioxid Redox Signal. 2013;19(4):415-30.

69. Lagouge M, Mourier A, Lee HJ, Spahr H, Wai T, Kukat C, et al. SLIRP regulates the rate of mitochondrial protein synthesis and protects LRPPRC from degradation. PLoS Genet. 2015;11(8):e1005423.

70. Shimakata T, Mihara K, Sato R. Reconstitution of hepatic microsomal stearoyl-coenzyme a desaturase system from solubilized components. J Biochem. 1972;72(5):1163-74.

71. Young RM, Ackerman D, Quinn ZL, Mancuso A, Gruber M, Liu L, et al. Dysregulated mTORC1 renders cells critically dependent on desaturated lipids for survival under tumor-like stress. Genes Dev. 2013;27(10):1115-31.

72. Gozzelino R, Arosio P. Iron homeostasis in health and disease. Int J Mol Sci. 2016;17:1. https://doi.org/10.3390/ijms17010130.

73. Frise MC, Cheng HY, Nickol AH, Curtis MK, Pollard KA, Roberts DJ, et al. Clinical iron deficiency disturbs normal human responses to hypoxia. J Clin Invest. 2016;126(6):2139-50.

74. Fulda S, Gorman AM, Hori O, Samali A. Cellular stress responses: cell survival and cell death. Int J Cell Biol. 2010;2010:214074

75. Takaishi S, Okumura T, Tu S, Wang SS, Shibata W, Vigneshwaran R, et al. Identification of gastric cancer stem cells using the cell surface marker CD44. Stem Cells. 2009;27(5):1006-20.

76. Commisso C, Davidson SM, Soydaner-Azeloglu RG, Parker SJ, Kamphorst J Hackett $\mathrm{S}$, et al. Macropinocytosis of protein is an amino acid supply route in Ras-transformed cells. Nature. 2013;497(7451):633-7.

77. Overmeyer $\mathrm{JH}$, Young AM, Bhanot $\mathrm{H}$, Maltese WA. A chalcone-related small molecule that induces methuosis, a novel form of non-apoptotic cell death, in glioblastoma cells. Mol Cancer. 2011;10:69. 4598-10-69
78. Palm W, Thompson CB. Nutrient acquisition strategies of mammalian cells Nature. 2017:546(7657):234-42.

79. Zaidi N, Lupien L, Kuemmerle NB, Kinlaw WB, Swinnen JV, Smans K. Lipogenesis and lipolysis: the pathways exploited by the cancer cells to acquire fatty acids. Prog Lipid Res. 2013;52(4):585-9.

80. Kamphorst JJ, Cross JR, Fan J, de Stanchina E, Mathew R, White EP, et al. Hypoxic and Ras-transformed cells support growth by scavenging unsaturated fatty acids from lysophospholipids. Proc Natl Acad Sci U S A. 2013;110(22):8882-7.

81. Ntambi JM. Regulation of stearoyl-CoA desaturase by polyunsaturated fatty acids and cholesterol. J Lipid Res. 1999:40(9):1549-58.

82. Ackerman D, Simon MC. Hypoxia, lipids, and cancer: surviving the harsh tumor microenvironment. Trends Cell Biol. 2014:24(8):472-8.

83. Merendino N, Costantini L, Manzi L, Molinari R, D'Eliseo D, Velotti F. Dietary omega -3 polyunsaturated fatty acid DHA: a potential adjuvant in the treatment of cancer. Biomed Res Int. 2013;2013(3):10186.

\section{Submit your next manuscript to BioMed Central and we will help you at every step:}

- We accept pre-submission inquiries

- Our selector tool helps you to find the most relevant journal

- We provide round the clock customer support

- Convenient online submission

- Thorough peer review

- Inclusion in PubMed and all major indexing services

- Maximum visibility for your research

Submit your manuscript at www.biomedcentral.com/submit
) Biomed Central 